\title{
Enhancing Photocatalytic Activity of NO Removal through an In Situ Control of Oxygen Vacancies in Growth of TiO2
}

Xiaoling Shen, Guohui Dong, Lan Wang, Liqun Ye and Jianwu Sun

The self-archived postprint version of this journal article is available at Linköping University Institutional Repository (DiVA):

$\underline{\text { http://urn.kb.se/resolve?urn=urn:nbn:se:liu:diva-159865 }}$

N.B.: When citing this work, cite the original publication.

Shen, X., Dong, G., Wang, L., Ye, L., Sun, J., (2019), Enhancing Photocatalytic Activity of NO Removal through an In Situ Control of Oxygen Vacancies in Growth of TiO2, Advanced Materials Interfaces, , 1901032. https://doi.org/10.1002/admi.201901032

Original publication available at:

https://doi.org/10.1002/admi.201901032

Copyright: Wiley (12 months)

http://eu.wiley.com/WileyCDA/ 


\section{WILEY-VCH}

DOI: 10.1002/ ((please add manuscript number))

Article type: (Full Paper)

\section{Enhancing Photocatalytic Activity of NO Removal Through an In-Situ Control of Oxygen Vacancies in Growth of $\mathrm{TiO}_{2}$}

Xiaoling Shen, Guohui Dong*, Lan Wang, Liqun Ye, and Jianwu Sun*

X. L. Shen, Dr. G. H. Dong, Dr. L. Wang,

School of Environmental Science and Engineering, Shaanxi University of Science and Technology, Xi'an 710021, P. R. China

E-mail: dongguohui@sust.edu.cn

Dr. L. Q. Ye

College of Materials and Chemical Engineering, Key Laboratory of Inorganic Nonmetallic Crystalline and Energy Conversion Materials, China Three Gorges University, Yichang 443002, P. R. China

Dr. J. W. Sun

Department of Physics, Chemistry and Biology, Linköping University, 58183 Linköping, Sweden

E-mail: jianwu.sun@liu.se

Keywords: Nitric oxide removal, oxygen vacancies, photocatalysis, $\mathrm{TiO}_{2}$

Although defects play an important role in the photocatalytic activity of $\mathrm{TiO}_{2}$, the mechanism of the photocatalytic activity related to different defects remains disputable. Moreover, the reported methods to introduce defects raise the preparation cost. In this work, different types of defects including O-vacancy cluster, surface O-vacancy and bulk O-vacancy defects were in-situ introduced in $\mathrm{TiO}_{2}$ by controlling the crystallization temperature. The medium-degree crystallinity $\mathrm{TiO}_{2}$ sample mainly containing surface O-vacancies exhibits the best $\mathrm{NO}$ removal activity. The systematic study of photocatalytic mechanism demonstrates that the surface Ovacancies significantly promote the adsorption of $\mathrm{H}_{2} \mathrm{O}$ molecules and improve charge transfer to the adsorbed $\mathrm{H}_{2} \mathrm{O}$ forming $\cdot \mathrm{OH}$, thus dramatically enhancing the photocatalytic $\mathrm{NO}$ removal activity. On the contrary, bulk O-vacancies neither help the adsorption of $\mathrm{H}_{2} \mathrm{O}$ molecules, nor improve the charge transfer to $\mathrm{H}_{2} \mathrm{O}$.

\section{Introduction}




\section{WILEY-VCH}

Titanium dioxide $\left(\mathrm{TiO}_{2}\right)$ was discovered as a photocatalytic material since the early part of 20th century. It is the most widely used photocatalyst because its low cost, strong oxidizing activity, high stability, and nontoxicity. ${ }^{[1-3]}$ However, the solar energy conversion efficiency of $\mathrm{TiO}_{2}$ is relatively low due to the following factors. Firstly, $\mathrm{TiO}_{2}$ with a large band gap of 3.0-3.2 eV can only absorb ultraviolet light, which is only about $4 \%$ in the solar spectrum. ${ }^{[4]}$ Secondly, the charge separation and transport in $\mathrm{TiO}_{2}$ are not efficient because of high recombination rates of photogenerated carriers. ${ }^{[5]}$ In order to solve these issues, various attempts such as noble metal deposition, construction of heterojunction, and foreign elements doping have been done. ${ }^{[6-8]}$ However, these methods would increase the cost of $\mathrm{TiO}_{2}$ photocatalysts.

It is reported that the introduction of defects such as $\mathrm{O}$ vacancy and $\mathrm{Ti}^{3+}$ could affect the photocatalytic activity of $\mathrm{TiO}_{2}$ due to two factors. ${ }^{[9,10]}$ First, defects may introduce energy levels in the band gap of $\mathrm{TiO}_{2}$ and the intermediate energy levels could enable the absorption of the sub-band gap photons, which enhance the harvesting of sunlight. Meanwhile, the intermediate energy levels induced by bulk defects might also trap photogenerated carriers and act as recombination centers. Second, the surface defects may induce energy band bending near the semiconductor surface, thus forming a built-in electric field near the surface that can greatly promote the separation and transport of the photogenerated electron-hole pairs. Generally, the type of defect depends on the defect construction method. It is reported that oxygen vacancy can be produced by re-calcination under the inert atmosphere. ${ }^{[11,12]}$ The formation of Ti-related defects needs special chemical (such as glycerol) was added in the precursors. ${ }^{[13]}$ Titanous $\left(\mathrm{Ti}^{3+}\right)$ can be created via hydrogenation treatment or $\mathrm{Ar}^{+}$ion sputtering. ${ }^{[14,15]}$ These methods inevitably raise the cost or the danger coefficient of $\mathrm{TiO}_{2}$ synthesis. As well-known, the crystallization course of $\mathrm{TiO}_{2}$ is a transition process from disordered state to ordered state. In fact, the nature of defects is the partial disorder in an ordered permutation of atoms. Therefore, the crystallization process of $\mathrm{TiO}_{2}$ is accompanied 


\section{WILEY-VCH}

with an evolution process of defects. We believe that a rational control of an existential state of defects in the synthesis process of $\mathrm{TiO}_{2}$ can significantly improve the photocatalytic activity of $\mathrm{TiO}_{2}$. Moreover, the introduction of defects in the synthesis process can reduce the fabrication cost. However, the mechanism of the photocatalytic activity related to different defects remains disputable. Therefore, the study of the photocatalytic mechanism of defects introduced in the synthesis process is highly critical to the design of efficient photocatalysts.

$\mathrm{NO}$ is one of the most common and harmful air pollutions for the ecological environment and human health because it can cause the acid rain, ozonosphere hole, greenhouse effect and photochemical $\operatorname{smog}^{[16]}$. Photocatalytic technology is regarded as an ideal method to remove low concentration NO in atmosphere. ${ }^{[17,18]}$ So far, the defective $\mathrm{TiO}_{2}$ have rarely been used for photocatalytic NO removal. In this study, we investigated the formation process of defects during the crystallization course of $\mathrm{TiO}_{2}$. Meanwhile, the NO removal activities of different $\mathrm{TiO}_{2}$ samples were studied. Our experiment results demonstrate that the vacancy clusters, surface oxygen vacancies, bulk oxygen vacancies can be respectively introduced as the main defects in different samples by controlling $\mathrm{TiO}_{2}$ crystallinity. Moreover, the medium-degree crystallinity $\mathrm{TiO}_{2}$ sample (m-350), which mainly contains surface oxygen vacancies, exhibits the best photocatalytic NO removal activity. The mechanism of the photocatalytic enhancement including the impacts of different defects on energy band, charge carrier transfer, and $\mathrm{H}_{2} \mathrm{O}$ molecular adsorption has been systematically studied.

\section{Results and Discussion}

\subsection{Structural Analysis}

X-ray diffraction (XRD) measurements were used to analyze the crystallinity and phase structure of different $\mathrm{TiO}_{2}$ samples. As shown in Figure S1a, the XRD pattern of m-0 has no obvious peaks, suggesting $\mathrm{m}-0$ is an amorphous structure. After calcinated at $350{ }^{\circ} \mathrm{C}$ for 4 


\section{WILEY-VCH}

hours, the XRD pattern of the sample m-350 shows a group of wide peaks, which could be indexed to anatase peaks (JCPDS file No.4-477). With further increasing the calcination temperature to $550{ }^{\circ} \mathrm{C}$, the anatase peaks in the XRD pattern of $\mathrm{m}-550$ become strong and sharp. This indicates that the crystallity of anatase $\mathrm{TiO}_{2}$ is increased with the increasing the calcination temperature. The XPS (Figure S1b and S1c) measurements confirm that samples $\mathrm{m}-0, \mathrm{~m}-350$, and $\mathrm{m}-550$ have identical chemical compositions (the detail description can be found in Supporting Information).

The high-resolution TEM (HRTEM) image and selected area electron diffraction (SAED) patterns were used to further investigate the crystallinity of three samples. From Figure S2b and S2c, $\mathrm{m}-0$ has no obvious lattice fringe and diffraction spot/ring, confirming $\mathrm{m}-0$ is amorphous $\mathrm{TiO}_{2}$. The HRTEM image of $\mathrm{m}-350$ shows many crystalline regions (Figure S2e and S2f). However, these crystalline regions are discontinuous and are separated by amorphous areas, indicating that $\mathrm{m}-350$ is microcrystal $\mathrm{TiO}_{2}$. When the calcination temperature was increased to $550{ }^{\circ} \mathrm{C}$, the HRTEM image of m-550 exhibits many crystalline regions (Figure S2h). Compared to $\mathrm{m}-350$, the crystalline regions in HRTEM image of $\mathrm{m}$ 550 became larger and coalesce with each other. Moreover, the SAED pattern of $\mathrm{m}-550$ has obvious diffraction spots (Figure S2i), suggesting the formation of polycrystal $\mathrm{TiO}_{2}$.

The crystallinity not only affects the morphology of sample, but also affects the defect states sample. To figure out the kind and position of defects in different samples, electron paramagnetic resonance (EPR) was firstly used to detect the presence of defects. As shown in Figure 1a, the EPR signal of $\mathrm{m}-0$ is very weak. After the calcination at $350{ }^{\circ} \mathrm{C}$ and $550{ }^{\circ} \mathrm{C}$, $\mathrm{m}-350$ and $\mathrm{m}-550$ displayed an obvious and symmetrical EPR signal at $\mathrm{g}=2.0024$ and $\mathrm{g}=$ 2.0026, respectively. Based on previous reports, both of these EPR signals can be assigned to the presence of oxygen vacancy. ${ }^{[19,20]}$ The subtle difference in $g$ factors is related to the different position of oxygen vacancy, which will be discussed in the section below. 


\section{WILEY-VCH}

Besides EPR spectra, positron annihilation spectroscopy (PLAS, Figure S3) was further used to analyze the defects in different samples. Table 1 shows two kinds of positron lifetimes $\tau_{1}$ and $\tau_{2}$, corresponding to two kinds of defects in samples. The smaller one $\left(\tau_{1}\right)$ is due to vacancy defects, while the larger one $\left(\tau_{2}\right)$ is generally caused by the presence of micro-pore defects. ${ }^{[21-23]}$ In $\mathrm{m}-0$, the concentration of $\tau_{2}$ is $66.3 \%$, suggesting the main defects in $\mathrm{m}-0$ are oxygen vacancy clusters. As for m-350 and m-550, the concentrations of $\tau_{1}$ in m-350 and m550 are $96 \%$ and $74 \%$, respectively. This indicates that the main defects in $m-350$ and $m-550$ are vacancy defects, confirming the EPR results that the defects in $m-350$ and $m-550$ are oxygen vacancies. But interestingly, the $\tau_{1}$ value of m-550 (0.29 ns) is smaller than that of m$350(0.32 \mathrm{~ns})$, implying the electron density in oxygen vacancy of m-350 is smaller than that of $\mathrm{m}-550 . \mathrm{In} \mathrm{TiO}_{2}$ structure, there have two possible $\mathrm{O}$ vacancies: surface $\mathrm{O}$ vacancy and bulk $\mathrm{O}$ vacancy (Figure 1b). Generally, the size of surface $\mathrm{O}$ vacancy is larger than that of bulk $\mathrm{O}$ vacancy. A larger size of $\mathrm{O}$ vacancy will lead to a lower electron density in oxygen vacancy. Therefore, the main defects in m-350 and m-550 are surface $\mathrm{O}$ vacancy and bulk $\mathrm{O}$ vacancy, respectively.

\subsection{Photocatalytic Activity Analysis}

According to previous reports, the better crystallinity of semiconductor will produce the stronger photocatalytic activity. ${ }^{[24,25]}$ However, this work demonstrates that $\mathrm{m}-550$, which has the best crystallization, shows the worst NO removal activity among three samples (Figure 2a). However, m-350 exhibits the best photocatalytic NO removal activity, which is nearly 10 times higher than that of $\mathrm{m}-550$ sample $\left(350{ }^{\circ} \mathrm{C}\right.$ is the optimal synthesis temperatures as shown in Figure S4a). The photocatalytic activity of m-350 is much better than that of commercial anatase $\left(\mathrm{CA}-\mathrm{TiO}_{2}\right)$ and $\mathrm{P} 25$, which is the famous commercial $\mathrm{TiO}_{2}$ (Figure 2b). The order of NO removal activity is $\mathrm{m}-350>\mathrm{m}-0>\mathrm{P} 25>\mathrm{CA}-\mathrm{TiO}_{2} \approx \mathrm{m}-550$. Based on above 


\section{WILEY-VCH}

analyses, the photocatalytic activities of the prepared samples firstly increase and then decrease with the improving of samples' crystallinity. This phenomenon suggests that crystallinity is not the main limiting fact for the photocatalytic NO removal in our case. To compare the activity of m-350 with similar samples, we constructed oxygen vacancy on the surface of m-550 (m-550-Ar), P25 (P25-Ar) and commercial anatase (CAT-Ar) by annealing them under the Ar gas flow at $250^{\circ} \mathrm{C}$ for $2 \mathrm{~h}$. After the NO removal experiments, we found that the NO removal activity of m-350 is much better than those of m-550-Ar, P25-Ar and CAT-Ar (Figure S4b), indicating the surface oxygen vacancy generated from the synthesis process may be better than those generated from the Post treatment.

Besides NO, the concentration of $\mathrm{NO}_{2}$ was also monitored. Meanwhile, the productions of $\mathrm{NO}_{3}{ }^{-}$and $\mathrm{NO}_{2}{ }^{-}$ion were investigated by ion chromatography (IC, ICS-90A, DIONEX). From Figure 2c, about $30 \mathrm{ppb}$ of $\mathrm{NO}_{2}$ could be detected in m-0 system after 30 min of light irradiation. However, $\mathrm{NO}_{2}$ could not be detected in $\mathrm{m}-350$ and $\mathrm{m}-550$ systems. Meanwhile, no $\mathrm{NO}_{2}{ }^{-}$productions could be found in three sample systems. From Figure 2d, the content of produced $\mathrm{NO}_{3}^{-}$in the $\mathrm{m}-0, \mathrm{~m}-350$ and $\mathrm{m}-550$ are $0.25,18.7$ and $0.85 \mu \mathrm{g} / \mathrm{L}$, respectively. According to the values of consumed $\mathrm{NO}$ and produced $\mathrm{NO}_{2}$ and $\mathrm{NO}_{3}{ }^{-}$, the $\mathrm{N}$ balances in three sample systems were calculated to determine whether other products were produced in the NO removal process (The calculation methods can be found in Supporting Information). The calculated results shown that the theoretical $\mathrm{NO}_{3}{ }^{-}$values in $\mathrm{m}-0, \mathrm{~m}-350$ and $\mathrm{m}-550$ systems were $4.34,19.84$ and $0.93 \mu \mathrm{g} / \mathrm{L}$, respectively. Obviously, the detected $\mathrm{NO}_{3}{ }^{-}$values of $\mathrm{m}-350$ and $\mathrm{m}-550$ approximately equal to their theoretical values, suggesting that $\mathrm{m}-350$ and m-550 can deeply oxidize $\mathrm{NO}$ to $\mathrm{NO}_{3}{ }^{-}$. However, in $\mathrm{m}-0$ system, the detected $\mathrm{NO}_{3}{ }^{-}$value was far less than its' theoretical value. It means that the NO removal in $\mathrm{m}-0$ system mainly resulted from adsorption besides small quantity of incomplete oxidation.

\subsection{Mechanism of the photocatalytic NO removal}




\section{WILEY-VCH}

The surface area might affect the catalytic activity of catalyst. Therefore, nitrogen adsorptiondesorption isotherms were employed to measure the surface areas of the samples discussed above. According to the detection results (Figure S5a), the Brunauer-Emmett-Teller (BET) specific surface areas of $\mathrm{m}-0, \mathrm{~m}-350$ and $\mathrm{m}-550$ are $246.278,49.688$ and $4.149 \mathrm{~m}^{2} \cdot \mathrm{g}^{-1}$, respectively, suggesting that the surface area of $\mathrm{TiO}_{2}$ in our systems is decreased with the increase of crystallinity. The surface area of $\mathrm{m}-0$ is much larger than those of $\mathrm{m}-350$ and $\mathrm{m}$ 550. This explains that the $\mathrm{NO}$ removal in $\mathrm{m}-0$ system is mainly resulted from adsorption. Although the surface area of $\mathrm{m}-350$ is only $1 / 5$ of that of $\mathrm{m}-0$, the produced $\mathrm{NO}_{3}{ }^{-}$in $\mathrm{m}-350$ system is 75 and 22 times higher than that in $\mathrm{m}-0$ and $\mathrm{m}-350$ system, respectively (Figure S5b). This result indicates that the significantly enhanced photocatalytic NO removal activity of $\mathrm{m}-350$ can not be explained in term of the increase of surface area.

Previous results proved that $\mathrm{O}$ vacancy has a significant effect on the photocatalytic activity of $\mathrm{TiO}_{2}{ }^{[26-30]} \mathrm{We}$ believe that the position of $\mathrm{O}$ vacancy in $\mathrm{TiO}_{2}$ could influence the $\mathrm{NO}$ adsorption, light absorption, charge generation and separation as well as carrier transport, thus determining the overall photocatalytic NO removal activity. To further study the mechanism of the enhanced photocatalytic activity, we first performed NO removal experiments under the dark condition to detect the NO adsorption quantity of different samples. As shown in Figure S6a, the NO concentration in m-0 system was first decreased by a quantity of $3.6 \mathrm{ppb}$ and then returned to a constant value without further decrease again. This indicates that the saturation of the NO adsorption on $\mathrm{m}-0$ surface has been reached, and the adsorbed $\mathrm{NO}$ could not desorb from the surface of $\mathrm{m}-0$ (Figure S6b). In the m-350 system, the adsorption quantity was only $1.3 \mathrm{ppb}$. After this weak adsorption, the concentration of NO periodically increases and decreases with time (Figure S6c), indicating that the adsorption and desorption of NO consistently occurred on the surface of m-350 (Figure S6d). The initial adsorption quantity of $\mathrm{m}-550$ is similar to that of $\mathrm{m}-350$. However, the decreased NO concentration in $\mathrm{m}-$ 


\section{WILEY-VCH}

550 system rises subsequently and decreases again after a long time, suggesting that the NO desorption from the surface of m-550 is difficult (Figure S6e and S6f).

To further study the NO adsorption ability of different samples, temperature-programmed desorption (TPD) experiments were used to compare the NO desorption energy of $\mathrm{m}-0, \mathrm{~m}-$ 350, and m-550 samples. As seen in Figure 3a, m-0 shows an asymmetric broad NO desorption peak above $160{ }^{\circ} \mathrm{C}$. The $\mathrm{m}-550$ sample exhibits a rather weak NO desorption peak, indicating that NO is difficult to be adsorbed on the well-crystallized sample m-550. Although NO can be adsorbed on the surface of amorphous $\mathrm{TiO}_{2}(\mathrm{~m}-0)$, the desorption energy of $\mathrm{NO}$ on the surface of $\mathrm{m}-0$ is at least $4.09 \mathrm{KJ} / \mathrm{mol}$ (The calculation process can be found in supporting information). Here, both the weak adsorption (m-550) and difficult desorption (m-0) of NO would decrease the photocatalytic activity of $\mathrm{NO}$ removal of $\mathrm{TiO}_{2}$. In m-350 system, the strength of NO desorption peak is intermediate between those of m-0 and m-550 samples. More importantly, the initial NO desorption temperature of $\mathrm{m}-350\left(65^{\circ} \mathrm{C}\right)$ is much smaller than those of m-0 and m-550 samples. Based on this temperature, the lowest desorption energy of NO on the surface of $\mathrm{m}-350$ is calculated to be $1.3 \mathrm{KJ} / \mathrm{mol}$. The appropriate strength and position of NO desorption peak suggest that NO can be easily adsorbed and desorbed on the surface of $\mathrm{m}-350$. This characteristic is beneficial to the process of the photocatalytic NO removal. As demonstrated above, the main defects in $m-350$ and $m-550$ are surface $O$ vacancy and bulk O vacancy, respectively. Therefore, we believe that the excellent performance of NO adsorption and desorption is caused by the surface oxygen vacancy of m-350 (Figure 3b).

Light is the energy source of the photocatalytic reaction. Therefore, the light absorption ability is an important characteristic that needs to be studied. The different color of three samples firstly implied that different samples have different light absorption ability (Figure 4a). UV-vis absorption spectra were used to investigate the light absorption ability of different samples. As shown in Figure 4b, the absorption spectrum of m-0 is consistent with that of $\mathrm{TiO}_{2}$ reported in previous references. ${ }^{[30]}$ After the calcination at $350{ }^{\circ} \mathrm{C}$ and $550{ }^{\circ} \mathrm{C}$, the 


\section{WILEY-VCH}

UV-vis absorption edges of $\mathrm{m}-350$ and $\mathrm{m}-550$ have a red shift of $30 \mathrm{~nm}$ compared with that of $\mathrm{m}-0$. This band gap shrinkage is most probably due to the presence of $\mathrm{O}$ vacancies. ${ }^{[31]}$ Our calculation shown below demonstrates that the $\mathrm{O} 2 \mathrm{p}$ orbitals mainly contribute to the conduction band (CB) minimum of $\mathrm{TiO}_{2}$. It is worthy to note that the absorption spectra of $m-350$ and $m-550$ in the visible region are different. m-550 has no light absorption in visible region, while m-350 has an obvious absorption in entire visible region from 400 to $800 \mathrm{~nm}$, which could not due to the absorption of $\mathrm{TiO}_{2}$ because of its large band gap of $3.13 \mathrm{eV}$ (Figure 4c). Thus, the absorption in the range of 400 to $800 \mathrm{~nm}$ relates to some kinds of auxochrome group on $\mathrm{m}-350$. The auxochrome group was proved to be surface hydroxyl group by infrared spectroscopy (Figure 4d). The presence of surface hydroxyl group is resulted from surface $\mathrm{O}$ vacancy of $\mathrm{m}-350$. This is because surface $\mathrm{O}$ vacancy can increase the surface energy of m-350 and the high surface energy makes m-350 adsorb more $\mathrm{H}_{2} \mathrm{O}$ molecular, which would form the surface hydroxyl group. Though $\mathrm{m}-550$ also has $\mathrm{O}$ vacancies in its structure, there has no absorption in entire visible region in the UV-vis absorption spectrum of $\mathrm{m}-550$. This confirms that the $\mathrm{O}$ vacancies in $\mathrm{m}-550$ are bulk $\mathrm{O}$ vacancies. Therefore, the presence of surface $\mathrm{O}$ vacancies is beneficial to adsorb the hydroxyl group, which is of vital importance for the excellent NO removal activity for sample m-350.

Photoluminescence (PL) measurements further confirm the presence of different types of defects in those samples. As shown in Figure 5a, m-0 has a significant fluorescence emission peak at $380.64 \mathrm{~nm}$ that due to the near-band-edge emission. ${ }^{[32]}$ The sample m-350 exhibits a broad emission peak around $400 \mathrm{~nm}$, which is probably related to the surface $\mathrm{O}$ vacancies (Figure 5b). The PL emission peak of sample m-550 is shifted to $420 \mathrm{~nm}$ and this emission might correspond to the radiative recombination via $\mathrm{O}$ vacancies in bulk (Figure $\mathbf{5 b}$ ).

The charge separation and transport were studied by surface photovoltage (SPV) measurements. As known, defects of semiconductors could affect the energy band of material and separate efficiency of charge, thus could affect the concentration of carriers on the surface 


\section{WILEY-VCH}

of material. Therefore, SPV could provide information on the efficiency of charge separation and transport. As shown in Figure 6a, the SPV spectrum of m-0 displayed a weak voltage signal which decays very quickly, indicating a very fast recombination of photogenerated electron-hole pairs in m-0 system. In contrast, the samples m-350 and m-550 show a much higher the photovoltage signals with much longer decay time than the sample m-0 (Figure $6 \mathbf{b}$ and 6c). This clearly demonstrates $m-350$ and $m-550$ have higher carrier lifetimes and thus larger carrier diffusion lengths, which gives rise higher photovoltages. Notably, m-550 displays a negative voltage signal before the positive voltage signal. This observation could be related to the fact that the defect energy levels in the band gap caused by bulk $\mathrm{O}$ vacancies first trap photogenerated carriers and then gradually release them. Moreover, the positive voltage signal of $m-350$ is smaller than that of $m-550$. The reason is that surface $O$ vacancy can improve the charge $\left(\right.$ holes $\mathrm{h}^{+}$) transfer from vacancy site to the adsorbate, and thus decrease the photovoltage of $\mathrm{m}-350$. This improvement of charge transfer via adsorbates can be further evidenced by the photocurrent measurement. As shown in Figure 6d the photocurrent density of $\mathrm{m}-350$ is much higher than that of $\mathrm{m}-550$, confirming surface $\mathrm{O}$ vacancy could promote the charge transfer ability of m-350.

The charge transfer from surface to the adsorbate is a critical step in the photocatalytic NO removal. This is because the redox half-reactions and the generation of active species occur in this stage. The type of occurred reaction and the generated active specie depend on the adsorption of adsorbates. The surface of $\mathrm{TiO}_{2}$ has either Ti or $\mathrm{O}$ terminal atoms (Schematic 1). During the $\mathrm{NO}$ adsorption, the possible adsorbates contain $\mathrm{NO}, \mathrm{O}_{2}, \mathrm{H}^{+}$and $\mathrm{OH}^{-}$. The nitrogen atom of NO has unpaired electron. It may be preferentially adsorbed on the terminal atom which has unpaired electron. Although both $\mathrm{O}$ and Ti terminations have single electrons, the electronegativity of $\mathrm{O}$ termination (3.5) is much large than that of Ti terminations (1.5). This demonstrates that $\mathrm{NO}$ would preferentially adsorb on the $\mathrm{O}$ termination of $\mathrm{TiO}_{2}$ through the covalent bonding. The $\mathrm{O}$ atoms of $\mathrm{O}_{2}$ and $\mathrm{OH}^{-}$have lone-pair electrons. This feature makes 


\section{WILEY-VCH}

them easy to bind with the terminal atom which has empty orbital through the formation of coordination bond. Ti termination of $\mathrm{TiO}_{2}$ has empty $3 \mathrm{p}$ orbital. Therefore, $\mathrm{O}_{2}$ and $\mathrm{OH}^{-}$ preferentially adsorb on $\mathrm{Ti}$ termination of $\mathrm{TiO}_{2}$. Since $\mathrm{H}^{+}$has a positive charge, it may prefer to bond with electronegative surface or atom. Therefore, compared with Ti termination, $\mathrm{H}^{+}$ prefers to adsorb at the $\mathrm{O}$ termination of $\mathrm{TiO}_{2}$.

The surface $\mathrm{O}$ vacancy can significantly affect the adsorbate of $\mathrm{m}-350$ (Figure 7a). The missing of surface $\mathrm{O}$ atoms would cause the increase of surface energy and the unbalance of surface charge. To restrain these changes, surface $\mathrm{O}$ vacancy favors to adsorb more hydroxyls. On the one hand, the radius of hydroxyl is similar with that of $\mathrm{O}$ atom. On the other hand, hydroxyl could balance the surplus positive charges. However, the hydroxyl in atmosphere is very small. This situation would make surface $\mathrm{O}$ vacancy adsorb more $\mathrm{H}_{2} \mathrm{O}$ molecules, which would ionize to more hydroxyls. Adsorption ability of $\mathrm{H}_{2} \mathrm{O}$ molecule can be investigated by the hydrophilic test as shown in Figure 7b-7d. Therefore, surface $\mathrm{O}$ vacancies facilitate the adsorption of electronegative hydroxyls, which results in a formation of band bending near the surface of $\mathrm{TiO}_{2}$, where the built-in electric field is directed from bulk to the surface. Upon light excitation, the photogenerated electron-hole pairs are separated by the built-in electric field. The hole would be swept to the surface for oxidation reaction while the electron would be swept to the opposite direction (Figure 7a).

According to the density of states (DOS) pattern, the valence band (VB) maximum of $\mathrm{TiO}_{2}$ is mainly constructed by Ti $3 \mathrm{~d}$ orbitals. $\mathrm{O} 2 \mathrm{p}$ orbitals contribute to the $\mathrm{CB}$ minimum of $\mathrm{TiO}_{2}$ (Figure 8a). In this case, photogenerated holes mainly produced at $\mathrm{Ti}$ atom, while photogenerated electrons mainly generate at $\mathrm{O}$ atom. Therefore, adsorbates which adsorbed at O termination would be reduced, while that adsorbed at Ti termination would be oxidized (Figure 8b). Although NO mainly adsorbed at $\mathrm{O}$ termination, it was not be reduced by the electrons because the products of NO removal were oxidation products. Based on this result, we conclude that the generated electrons during the NO removal process were mainly used to 


\section{WILEY-VCH}

reduce $\mathrm{H}^{+}$. This conclusion can be confirmed by the $\mathrm{H}_{2}$ evolution experiments in the next paragraph. Although $\mathrm{O}_{2}$ is mainly adsorbed on Ti termination, it cannot be oxidized by the holes. Meanwhile, the VB potential is positive than the redox potential of $\cdot \mathrm{OH} / \mathrm{OH}^{-}(2.27 \mathrm{eV}$, Figure 9a, Figure S7), suggesting that photogenerated holes were used to oxidize surface hydroxyl group to produce $\cdot \mathrm{OH}$. In $\mathrm{m}-350$, photogenerated holes are mainly captured by surface $\mathrm{O}$ vacancy, which adsorbed a lot of hydroxyls beforehand. Therefore, the surface $\mathrm{O}$ vacancy would make the production of $\cdot \mathrm{OH}$ in $\mathrm{m}-350$ system more easily. This can be proved by the detection of hydroxyl radicals, which will be described in the later section.

To confirm the reduction of $\mathrm{H}^{+}$by electrons generated during $\mathrm{NO}$ removal, we measure the $\mathrm{H}_{2}$ evolution for the three samples under the Xe lamp (300 W) irradiation. As shown in Figure 9b, $\mathrm{m}-0, \mathrm{~m}-350$, and $\mathrm{m}-550$ samples show the evolution of $\mathrm{H}_{2}$ under the light irradiation. After 2 hours of reaction, the amount of generated $\mathrm{H}_{2}$ in $\mathrm{m}-0, \mathrm{~m}-350$, and $\mathrm{m}-550$ systems were $0.14,11.5$, and $1.03 \mathrm{mmol} / \mathrm{g}$, respectively. The order of $\mathrm{H}_{2}$ evaluation activity is $\mathrm{m}-350>\mathrm{m}-550>\mathrm{m}-0$. This confirms that the electrons generated during NO removal can reduce $\mathrm{H}^{+}$to $\mathrm{H}_{2}$. The excellent consumption of electrons in the reduction half-reaction would significantly promote the half-reaction of the $\mathrm{OH}^{-}$oxidation.

In order to further investigate the oxidizing half-reaction, we measured the hydroxyl radicals $(\cdot \mathrm{OH})$ under aerobic and anaerobic conditions (Figure 9c and 9d). Under both aerobic and anaerobic conditions, there have no $\cdot \mathrm{OH}$ was produced in the $\mathrm{m}-0$ system. The reason may be that very few $\mathrm{H}_{2} \mathrm{O}$ can be adsorbed on the surface of m- 0 . Unlike $\mathrm{m}-0$, both m350 and $\mathrm{m}-550$ can produce $\cdot \mathrm{OH}$ under both aerobic and anaerobic conditions. However, the produced $\cdot \mathrm{OH}$ under anaerobic condition is more than that under aerobic condition, confirming that $\cdot \mathrm{OH}$ in $\mathrm{m}-350$ and $\mathrm{m}-550$ systems was mainly produced via the oxidation process. Moreover, the produced $\cdot \mathrm{OH}$ amount in $\mathrm{m}-350$ system is much more than that in $\mathrm{m}-$ 550 system. This is due to both the efficient capture of hole by surface $\mathrm{O}$ vacancy and the high adsorbed ability of $\mathrm{H}_{2} \mathrm{O}$ molecular on the surface of m-350. 


\section{WILEY-VCH}

Since the products of NO removal are mainly the oxidation products, $\cdot \mathrm{OH}$ or $\mathrm{h}^{+}$may play major role in NO removal process of three samples. In order to confirm this conclusion, tertbutanol (TBA) and potassium iodide (KI) were chosen as $\cdot \mathrm{OH}$ and $\mathrm{h}^{+}$scavengers, respectively. In both $\mathrm{m}-0$ and $\mathrm{m}-550$ systems, the trapping of $\cdot \mathrm{OH}$ did not affect the NO removal performance of $\mathrm{m}-0$ and $\mathrm{m}-550$ (Figure S8a and S8c). This is consistent with the fact that both $\mathrm{m}-0$ and $\mathrm{m}-550$ could not produce $\cdot \mathrm{OH}$ in gas-solid phase condition (Figure S8d and S8f). However, the capture of $\mathrm{h}^{+}$significantly inhibited the NO removal of $\mathrm{m}-0$ and $\mathrm{m}-550$, suggesting that the NO removal of $\mathrm{m}-0$ and $\mathrm{m}-550$ were mainly contributed by $\mathrm{h}^{+}$. The NO removal of $\mathrm{m}-0$ and $\mathrm{m}-550$ can be expressed by the equation 4 . In $\mathrm{m}-350$, the trapping of both $\cdot \mathrm{OH}$ and $\mathrm{h}^{+}$could significantly inhibit the NO removal of $\mathrm{m} \cdot-350$ (Figure $\mathbf{S 8 b}$ ). This is consistent with the above result that $\cdot \mathrm{OH}$ in $\mathrm{m}-350$ system mainly produced via the oxidation of $\mathrm{h}^{+}$. Meanwhile, $\mathrm{m}-350$ could produce $\cdot \mathrm{OH}$ in gas-solid phase condition (Figure S8e). Therefore, the NO removal of m-350 can be expressed by the equation 3 and 5 .

$$
\begin{aligned}
& \mathrm{TiO}_{2}+\text { light } \rightarrow \mathrm{e}^{-}+\mathrm{h}^{+} \\
& 2 \mathrm{e}^{-}+2 \mathrm{H}^{+} \rightarrow \mathrm{H}_{2} \\
& \mathrm{~h}^{+}+\mathrm{OH}^{-} \rightarrow \cdot \mathrm{OH} \\
& 3 \mathrm{~h}^{+}+\mathrm{NO}+2 \mathrm{H}_{2} \mathrm{O} \rightarrow \mathrm{NO}_{3}{ }^{-}+4 \mathrm{H}^{+} \\
& 3 \cdot \mathrm{OH}+\mathrm{NO} \rightarrow \mathrm{NO}_{3}^{-}+\mathrm{H}_{2} \mathrm{O}+\mathrm{H}^{+}
\end{aligned}
$$

Based on the results of produced $\mathrm{NO}_{3}{ }^{-}$and reaction mechanism, the apparent quantum yield $(\psi)$ of $\mathrm{m}-0, \mathrm{~m}-350$ and $\mathrm{m}-550$ were calculated as $0.91 \%, 68.33 \%$ and $3.11 \%$ respectively (The calculation process can be found in Supporting Information). Compared with m-0, m-350 has excellent stability to removal NO (Figure S9a). The slight decrease of the activity of 350 after 5 cycles is because the adsorbed $\mathrm{NO}_{3}^{-}$. When these $\mathrm{NO}_{3}^{-}$are washed by water, the activity of m-350 can recover (Figure S9b). Moreover, the XRD pattern, UV-vis absorption spectrum, and EPR pattern of the m-350 sample after photocatalytic measurements are identical to those of the pristine $\mathrm{m}-350$, indicating that the structure and surface $\mathrm{O}$ vacancies 


\section{WILEY-VCH}

of m-350 are stable (Figure S10a-S10c). The excellent quantum yield and stability indicate that $\mathrm{m}-350$ has strong practicability, which is depends on the in-situ generated surface oxygen vacancy in the growth of $\mathrm{TiO}_{2}$.

\section{Conclusion}

In this study, we investigated the different types of defects created during the crystallization process of $\mathrm{TiO}_{2}$ and their effects on the photocatalytic removal of NO. By controlling the calcination temperature, we successfully synthesized three types of $\mathrm{TiO}_{2}$ samples with a low-, medium-, and high-degree crystallinity. The main defects in these three types of $\mathrm{TiO}_{2}$ samples are oxygen vacancy cluster, surface oxygen vacancy and bulk oxygen vacancy defects, respectively. The $\mathrm{TiO}_{2}$ sample with a medium-degree crystallinity, which mainly contains surface oxygen vacancy defects, exhibits the best photocatalytic NO removal activity among all samples. While the $\mathrm{TiO}_{2}$ sample with a high-degree crystallinity, which mainly contains bulk oxygen vacancy defects, shows the worst photocatalytic NO removal activity. The mechanism of the enhanced photocatalytic NO removal activity is systematically investigated. We demonstrate that the surface $\mathrm{O}$ vacancies significantly promote the adsorption of $\mathrm{H}_{2} \mathrm{O}$ molecules and improve charge transfer to the adsorbated $\mathrm{H}_{2} \mathrm{O}$ forming $\cdot \mathrm{OH}$, thus dramatically enhancing the photocatalytic NO removal activity. On the contrary, bulk O vacancies neither help the adsorption of $\mathrm{H}_{2} \mathrm{O}$ molecules, nor improve the charge transfer to $\mathrm{H}_{2} \mathrm{O}$. This work provides a facile, secure and low-cost method to significantly improve the photocatalytic activity of $\mathrm{TiO}_{2}$. Moreover, the mechanism of the surface defects for improvement of the photocatalytic activity would shed light on design of highly efficient semiconductor photocatalysts.

\section{Experimental Section}




\section{WILEY-VCH}

Preparation of Photocatalysts: To prepare $\mathrm{TiO}_{2}$ nanoparticles, $5 \mathrm{ml}$ Tetrabutyl titanate (TBOT, analytical purity) was first slowly added into a $100 \mathrm{ml}$ beaker containing $50 \mathrm{ml}$ deionized water. Then, this beaker was silently placed for 10 hours without stirring. After this standing, the mixture was dried at $60{ }^{\circ} \mathrm{C}$ after being washed thoroughly by absolute ethyl alcohol and deionized water. Finally, the dried precursor was calcinated in a muffle furnace for $4 \mathrm{~h}$ at $350{ }^{\circ} \mathrm{C}$ and $550{ }^{\circ} \mathrm{C}$, respectively. After the calcination, the samples calcined at different temperature were denoted as $\mathrm{m}-350$ and $\mathrm{m}-550$. For comparison, the dried precursor without calcination was denoted as the sample $\mathrm{m}-0$.

Sample Characterization: The crystal structure of the samples was investigated by a D8 Advance X-ray diffractmeter (XRD) using $\mathrm{Cu} \mathrm{K \alpha}$ radiation under the scan speed of $4^{\circ} / \mathrm{min}$. The micro-morphology and structure of samples were measured by transmission electron microscope (TEM, JEOL 200kV). Surface areas of different samples were detected by a nitrogen adsorption desorption instrument (ASAP 2460). The temperature-programmed NO desorption (TPD) of different samples was measured by a Chemical adsorption instrument (PCA-1200). The light absorbance spectra of different samples were measured by a UV-2600 spectrophotometer (Shimadzu). Emission spectra of different samples were detected by fluorescence spectrophotometer (F-7000, Hitachi) under the excitation wavelength at $364 \mathrm{~nm}$. The elements composition and valence states of different samples were investigated in an Xray photoelectron spectroscopy (XPS, AXIS SUPRA, UK). Electron paramagnetic resonance spectrometer (EPR, Bruker A300) was employed to study defect states of different samples. Photocatalytic NO Removal Test: In the NO removal experiments, a sample dish (Radius = 3 $\mathrm{cm}$ ) containing $30 \mathrm{mg}$ of samples was placed in the middle of the glass columniform reactor $($ Radius $=4 \mathrm{~cm}$; height $=10 \mathrm{~cm})$ with a quartz skylight. An air stream containing $600 \mathrm{ppb}$ of NO continually flows through $(1 \mathrm{~L} / \mathrm{min})$ the surface of samples. Meanwhile, a $300 \mathrm{~W}$ xenon lamp with a $420 \mathrm{~nm}$ band pass filter as the visible light source, and vertically placed above the quartz skylight of the reactor. After the equilibrium was reached between NO and samples, 


\section{WILEY-VCH}

the light was turned on until the concentration of NO reduced to the minimum value. During the whole process, the NO concentration was monitored by a NOx analyzer (42i, Thermo Scientific).

\section{Supporting Information}

Supporting Information is available from the Wiley Online Library or from the author.

\section{Acknowledgements}

Financial support by the National Nature Science Foundation of China (Grant Nos. 21876104, 21603271, U1703129 and 11875248) are gratefully appreciated. J.W.S. acknowledges the Swedish Research Council (Vetenskapsrådet, Grant No. 621-2014-5461; 2018-04670), the Swedish Research Council for Environment, Agricultural Sciences and Spatial Planning (FORMAS, Grant No. 201600559), and the Swedish Foundation for International Cooperation in Research and Higher Education (STINT, Grant No. CH2016-6722). The authors are grateful to Mr. Run Ye and Prof. Bangjiao Ye from the State Key Laboratory of Particle Detection and Electronics (University of Science and Technology of China) for their helps with PALS measurements.

Received: ((will be filled in by the editorial staff))

Revised: ((will be filled in by the editorial staff)) Published online: ((will be filled in by the editorial staff))

\section{References}

[1] P. V. Kamat, J. Phys. Chem. C. 2012, 116, 11849.

[2] L. Zhao, X. F. Chen, X. C. Wang, Y. J. Zhang, W. Wei, Y. H. Sun, M. Antonietti, M.

M. Titirici, Adv. Mater. 2010, 22, 3317.

[3] J. Du, X. Y. Lai, N. L. Yang, J. Zhai, D. Kisailus, F. B. Su, D. Wang, L. Jiang, $A C S$

Nano. 2011, 5, 590.

a) J. Tao, T. Luttrell, M. Batzill, Nat. Chem. 2011, 3, 296. b) G. Liu, Y. N. Zhao, C. H.

Sun, F. Li, G. Q. Lu, H. M. Cheng, Angew. Chem. Int. Ed. 2008, 47, 4516. c) J. Tao, T. Luttrell, M. Batzill, Nat. Chem. 2011, 3, 296.

[5] C. A. Caputo, L. D. Wang, R. Beranek, E. Reisner, Chem Sci. 2015, 6, 5690. 


\section{WILEY-VCH}

[6] a) M. D. Ye, J. J. Gong, Y. K. Lai, C. J. Lin, Z. Q. Lin, J. Am. Chem. Soc. 2012, 134, 15720. b) J. W. Wan, W. X. Chen, C. Y. Jia, L. R. Zheng, J. C. Dong, X. S. Zheng, Y. Wang, W. S. Yan, C. Chen, Q. Peng, D. S. Wang, Y. D. Li, Adv. Mater. 2018, 30, 1705369.

[7] a) J. Q. Yan, G. J. Wu, N. J. Guan, L. D. Li, Appl. Catal. B: Environ. 2014, 152-153, 280. b) Y. Q. Zhu, M. W. Shah, C. Y. Wang. Appl. Catal. B: Environ. 2017, 203, 526. c) X. W. Li, W. D. Zhang, W. Cui, J. Y. Li, Y. J. Sun, G. M. Jiang, H. W. Huang, Y. X. Zhang, F. Dong. Chem. Eng. J. 2019, 370, 1366.

[8] a) R. Asahi, T. Morikawa, H. Irie, T. Ohwaki, Chem. Rev. 2014, 114, 9824. b) X. L. Xing, H. H. Zhu, M. Zhang, L. L. Hou, Q. Y. Li, J. J. Yang, Catal. Sci. Technol. 2018, 8, 3629. c) Y. N. Xin, Y. Huang, K. Lin, Y. F. Yu, B. Zhang. Sci. Bull, 2018, 63, 601.

[9] a) F. Zuo, K. Bozhilov, R. T. Dillon, L. Wang, P. Smith, X. Zhao, C. Bardeen, P. Y. Feng, Angew. Chem. Int. Ed. 2012, 51, 6223. b) X. B. Chen, L. Lei, P. Y. Yu, S. S. Mao, Science. 2011, 331, 746. c) J. Cao, Y. Zhang, L. Liu, J. Ye, Chem. Commun. 2013, 49, 3440.

[10] a) Z. C. Lian, W. C. Wang, G. S. Li, F. H. Tian, K. S. Schanze, H. X. Li, ACS Appl. Mater. Interfaces, 2017, 9, 16959. b) P. Niu, L. C. Yin, Y. Q. Yang, G. Liu, H. M. Cheng, Adv. Mater. 2014, 26, 8046. c) H. Li, F. Qin, Z. P. Yang, X. M. Cui, J. F. Wang, L. Z. Zhang, J. Am. Chem. Soc. 2017, 139, 3513.

[11] S. B. Wang, L. Pan, J. J. Song, W. B. Mi, J. J. Zou, L. Wang, X. J. Zhang, J. Am. Chem. Soc. 2015, 137, 2975.

[12] J. Coey, M. Venkatesan, C. Fitzgerald, Nat. Mater. 2005, 4, 173.

[13] M. K. Nowotny, L. R. Sheppard, T. Bak, J. Nowotny, J. Phys. Chem. C. 2008, 112, 5275.

[14] X. Yu, B. Kim, Y. K. Kim, ACS Catal. 2013, 3, 2479.

[15] a) X. D. Jiang, Y. P. Zhang, J. Jing, Y. S. Rong, Y. C. Wang, Y. C. Wu, C. X. Pan, J. Phys. Chem. C. 2015, 116, 22619. b) C. Y. Mao, F. Zuo, Y. Hou, X. H. Bu, P. Y. Feng, Angew. Chem. Int. Ed. 2014, 53, 10485. 


\section{WILEY-VCH}

[16] J. Y. Li, X. A. Dong, G. Zhang, W. Cui, W. L. Cen, Z. B. Wu, S. C. Lee, F. Dong. J. Mater. Chem. A, 2019, 7, 3366.

[17] a) H. Li, H. Shang, X. M. Cao, Z. P, Yang, Z. H. Ai, L. Z. Zhang, Environ. Sci. Technol. 2018, 52, 8659. b) G. H. Dong, D. Jacobs, L. Zang, C. Y. Wang, Appl. Catal. B: Environ, 2017, 218, 515.

[18] a) G. H. Dong, L. L. Zhao, X. X. Wu, M. S. Zhu, F. Wang, Appl. Catal. B: Environ 2019, 245, 459. b) T. Xiong, W. L. Cen, Y. X. Zhang, F. Dong, ACS Catal. 2016, 6, 2462. c) Z. H. Ai, W. K. Ho, S. C. Lee, L. Z. Zhang, Environ. Sci. Technol. 2009, 43, 4143.

[19] N. Kumar, U. Maitra, V. Hegde, U. Waghmare, A. Sundaresan, C. Rao, Inorg. Chem. 2013, 52, 10512 .

[20] S. Ghosh, G. Khan, K. Mandal, A. Samanta, P. Nambissan, J. Phys. Chem. C. 2013, 117,8458 .

[21] Y. Q. Yang, L. C. Yin, Y. Gong, P. Niu, J. Q. Wang, L. Gu, X. B. Chen, G. Liu, L. Z. Wang, H. M. Cheng, Adv. Mater. 2018, 30, 1704479.

[22] M. Kong, Y. Z. Li, X. Chen, T. T. Tian, P. F. Fang, F. Zheng, X. J. Zhao, J. Am. Chem. Soc. 2011, 133, 16414.

[23] J. Q. Yan, G. J. Wu, N. J. Guan, L. D. Li, X. Z. Cao, Phys. Chem. Chem. Phys, 2013, $15,10978$.

[24] W. Zhou, F. F. Sun, K. Pan, G. H. Tian, B. J. Jiang, Z. Y. Ren, C. G. Tian, H. G. Fu, Adv. Funct. Mater. 2011, 21, 1922.

[25] G. H. Tian, H. G. Fu, L. Q. Jing, C. G. Tian, J. Hazard. Mater. 2009, 161, 1122.

[26] C. C. Li, T. Wang, Z. J. Zhao, W. M. Yang, J. F. Li, A. Li, Z. L. Yang, G. Ozin, J. L. Gong, Angew. Chem. Int. Ed. 2018, 57, 5278.

[27] A. Naldoni, M. Allieta, S. Santangelo, M. Marelli, F. Fabbri, S. Cappelli, C. Bianchi, R. Psaro, V. Santo, J. Am. Chem. Soc. 2012, 134, 7600. 


\section{WILEY-VCH}

[28] H. Zhang, J. M. Cai, Y. T. Wang, M. Q. Wu, M. Meng, Y. Tian, X. G. Li, J. Zhang, L. R. Zheng, Z. Jiang, J. L. Gong, Appl. Catal. B: Environ. 2018, 434, 126.

[29] Q. P. Wu, F. Huang, M. S. Zhao, J. Xu, J. C. Zhou, Y. S. Wang, Nano Energy, 2016, 24,63 .

[30] W. J. Li, Y. T. Chen, P. H. Huang, T. L. Yang, J. J. Huang, J. Biophotonics. 2017, 10, 92.

[31] a) B. J. Morgan, G. W. Watson, J. Phys. Chem. C. 2010, 114, 2321. b) X. M. Yu, B. Kim, Y. K. Kim, ACS Catal. 2013, 3, 2479. c) Y. H. Zhang, R. Y. Dai, S. R. Hu, Phys. Chem. Chem. Phys. 2017, 19, 7307.

[32] a) H. Song, C. X. Li, Z. R. Lou, Z. Z. Ye, L. P. Zhu, ACS Sustainable Chem. Eng. 2017, 5, 8982. b) L. Hou, Z. Guan, M. Zhang, C. He, Q. Li, J. Yang, Catal. Sci. Technol. 2018, 10, 2809. 


\section{WILEY-VCH}

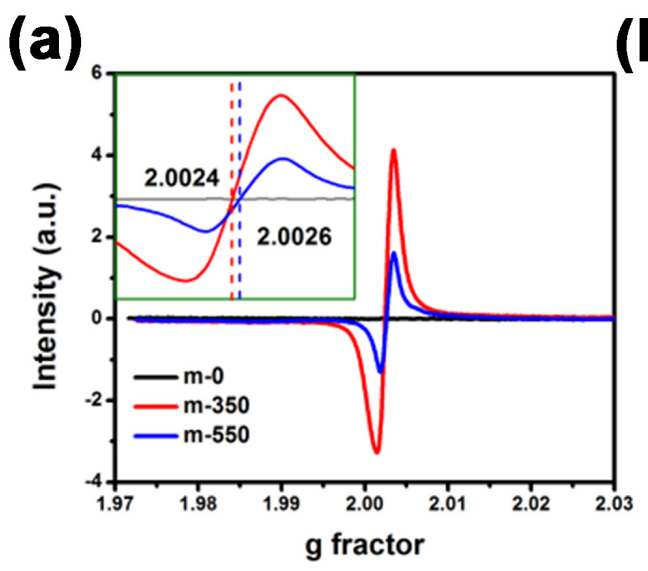

(b)

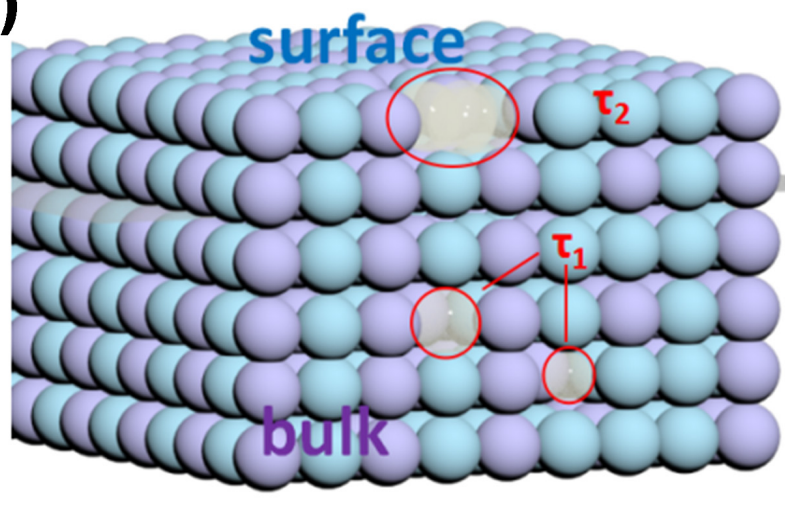

Figure 1. (a) The EPR signals of $\mathrm{TiO}_{2}$ samples without $(\mathrm{m}-0)$ and with calcination at $350{ }^{\circ} \mathrm{C}$ $(\mathrm{m}-350)$ and $550{ }^{\circ} \mathrm{C}(\mathrm{m}-550)$; (b) the diagram of surface oxygen vacancy and bulk oxygen vacancy in $\mathrm{TiO}_{2}$ structure.
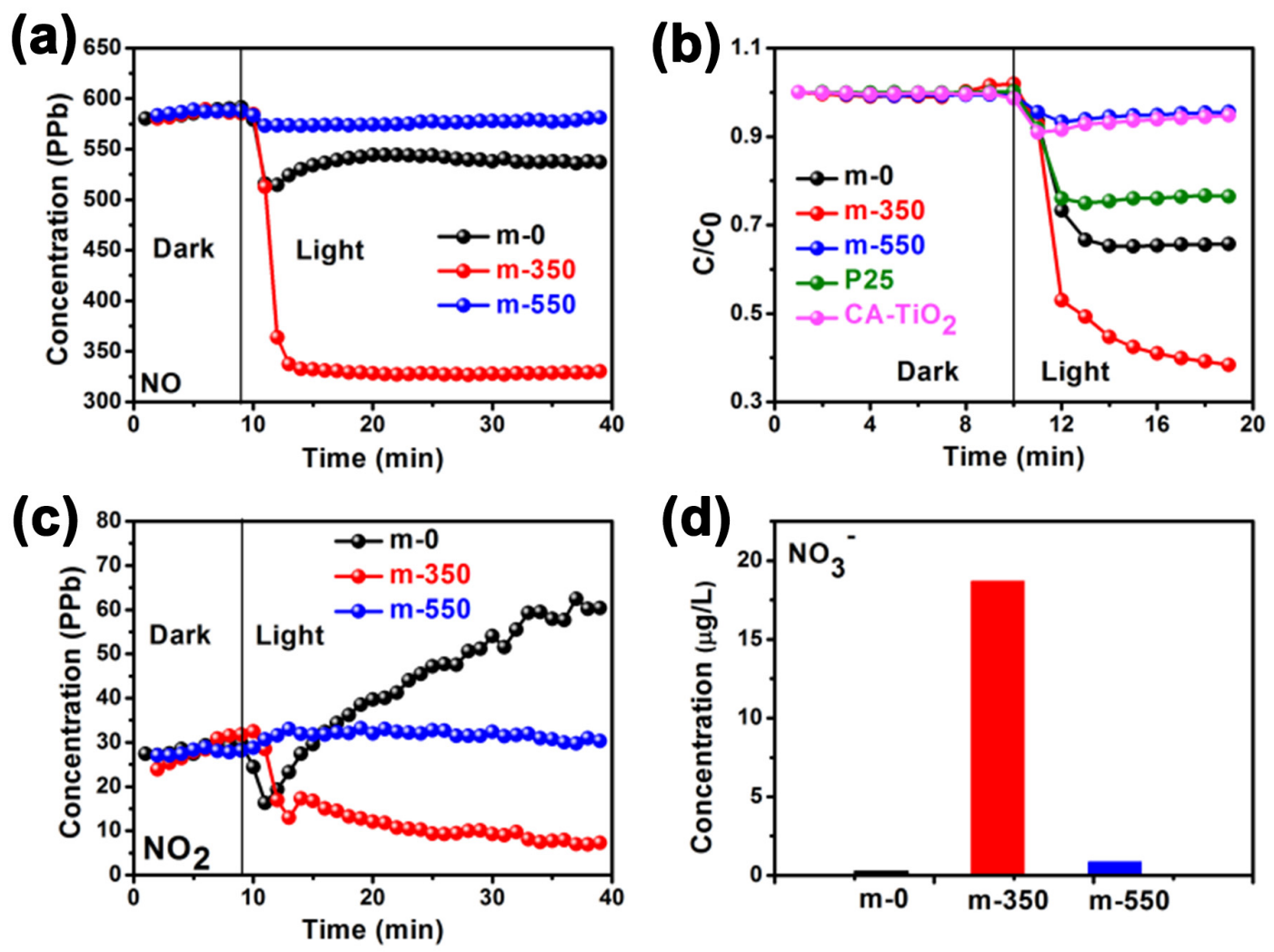

Figure 2. Photocatalytic NO removal activities of m-0, m-350, and $m-550$ (a); the activity comparison between the prepared samples and commercial $\mathrm{TiO}_{2}(\mathrm{~b})$; the concentration of $\mathrm{NO}_{2}$ in the $\mathrm{NO}$ removal process (c); the concentration of $\mathrm{NO}_{3}{ }^{-}$in the $\mathrm{NO}$ removal process (d). 


\section{WILEY-VCH}
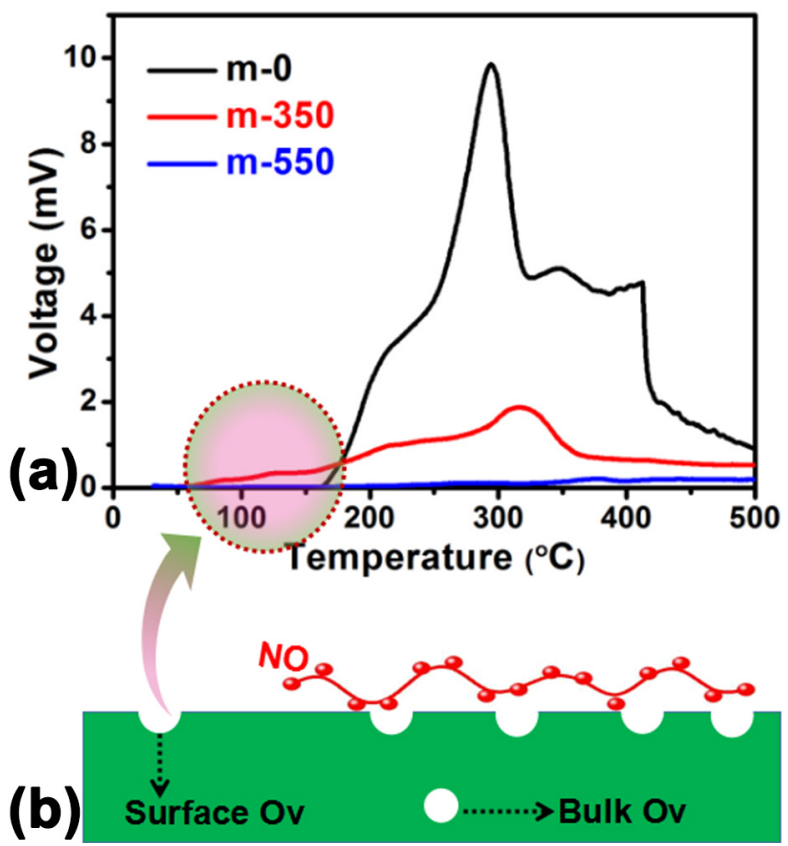

Figure 3.Temperature-programmed NO desorption curves of $m-0, m-350$, and $m-550$ (a); the excellent performance of $\mathrm{NO}$ adsorption and desorption is caused by the surface oxygen vacancy of $\mathrm{m}-350$ (b).

(a)

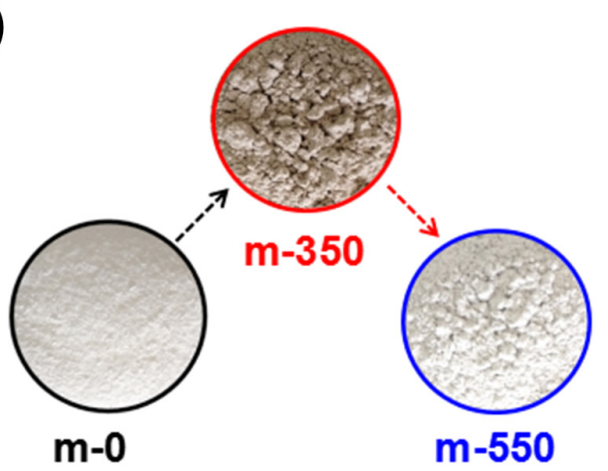

(c)

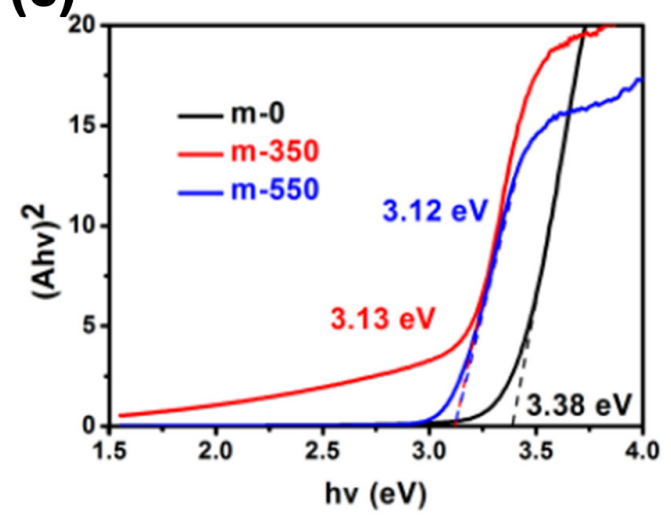

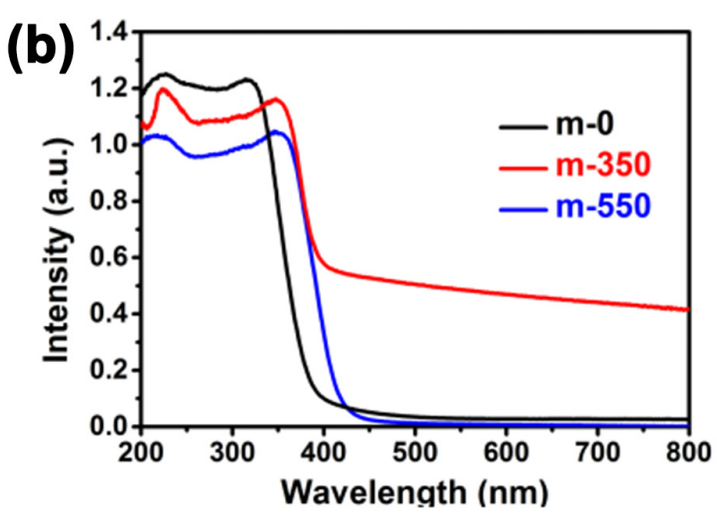

(d)

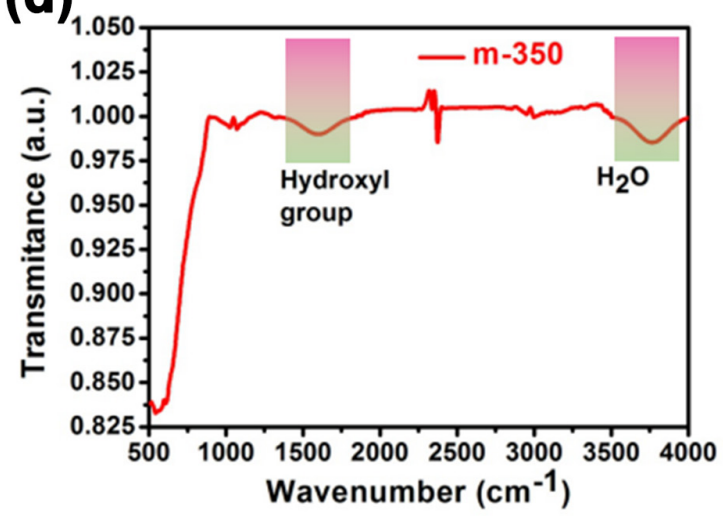

Figure 4. The photographs of different samples (a); UV-vis absorption spectra of different samples (b); the plots of (ahv) ${ }^{2}$ versus energy (hv) of different samples (c); infrared spectrum of $m-350$ (d). 


\section{WILEY-VCH}

(a)

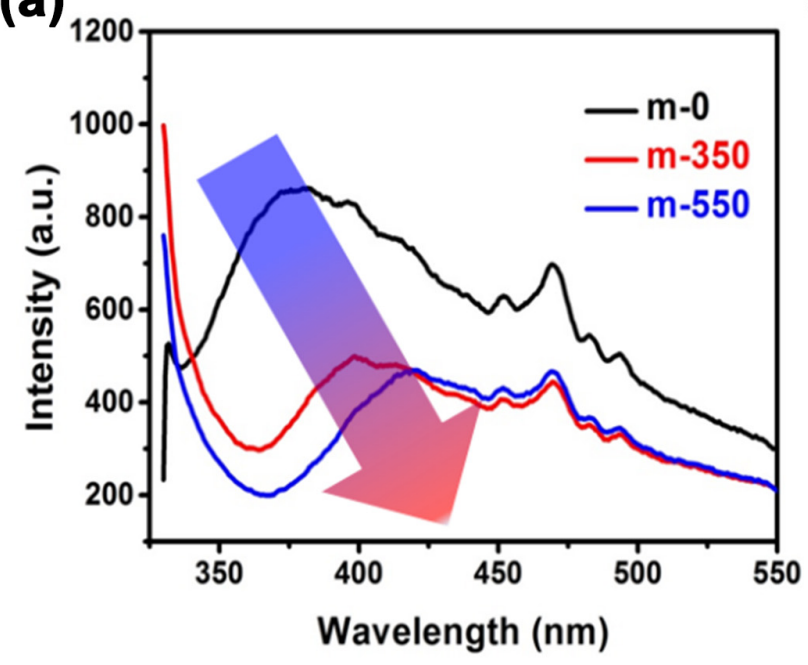

(b)

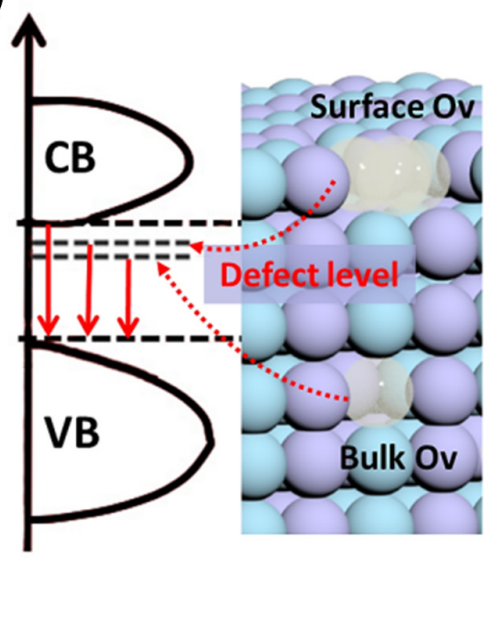

Figure 5. Photoluminescence (PL) spectra of different samples (a); the schematic diagram of the reason of red shift in PL spectra (b).

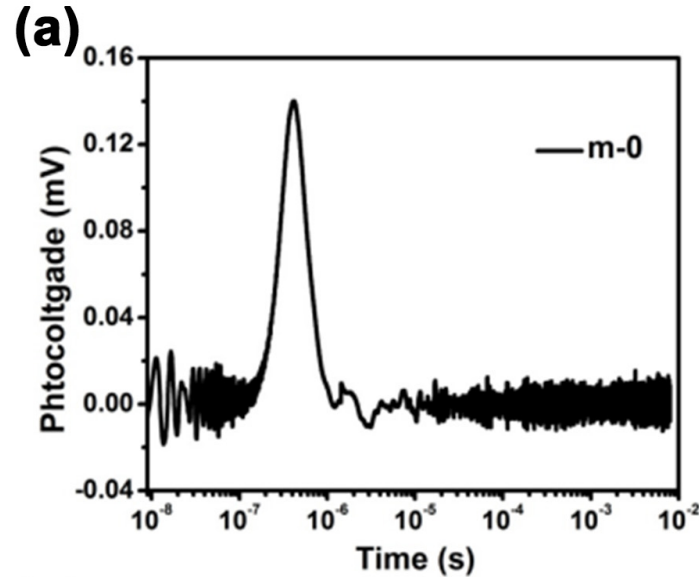

(c)

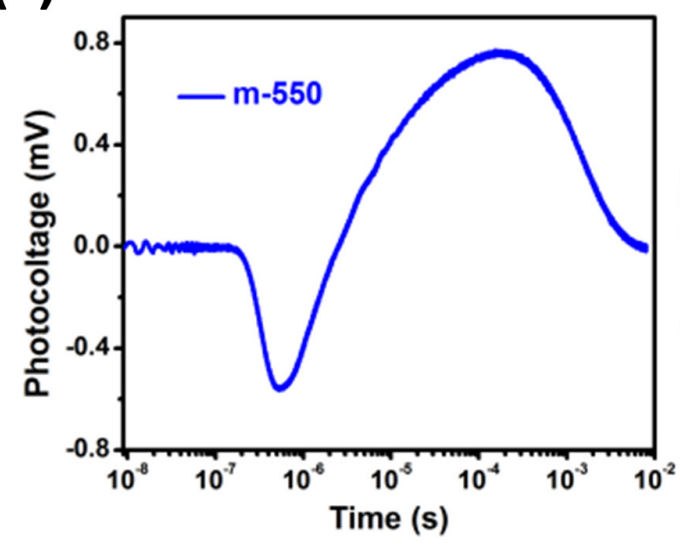

(b)

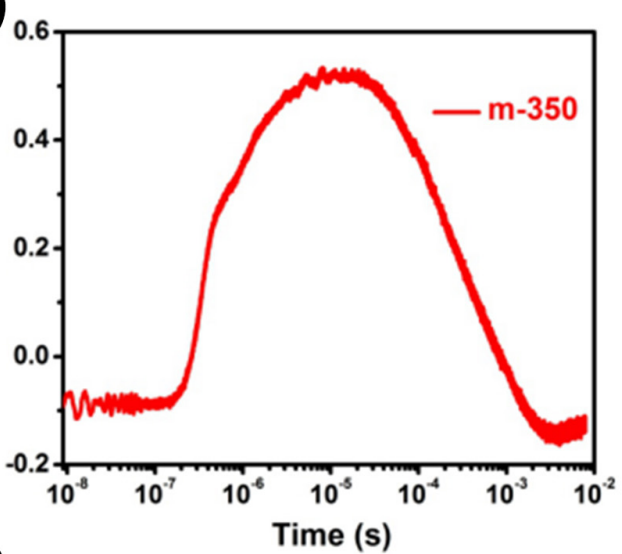

(d)

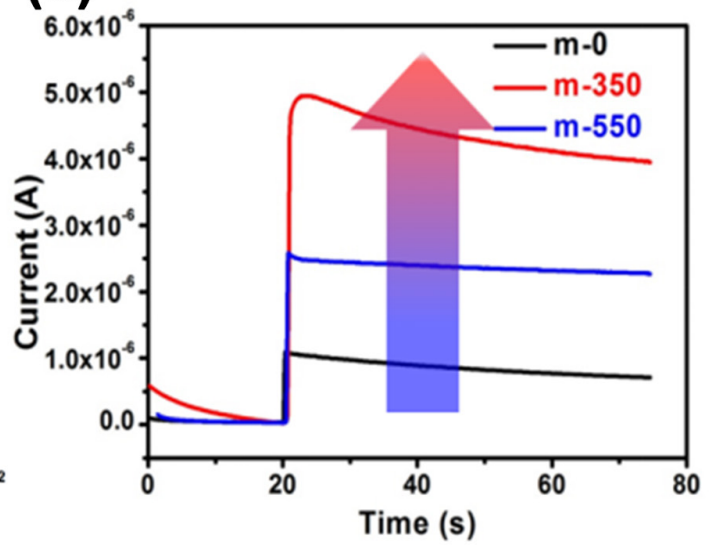

Figure 6. Surface photovoltage spectra of m-0 (a), m-350 (b), and m-550 (c); Photocurrent curves of different samples (d). 


\section{WILEY-VCH}

(a) O vacancy $\mathrm{H}_{2} \mathrm{O} \odot \mathrm{OH}-$
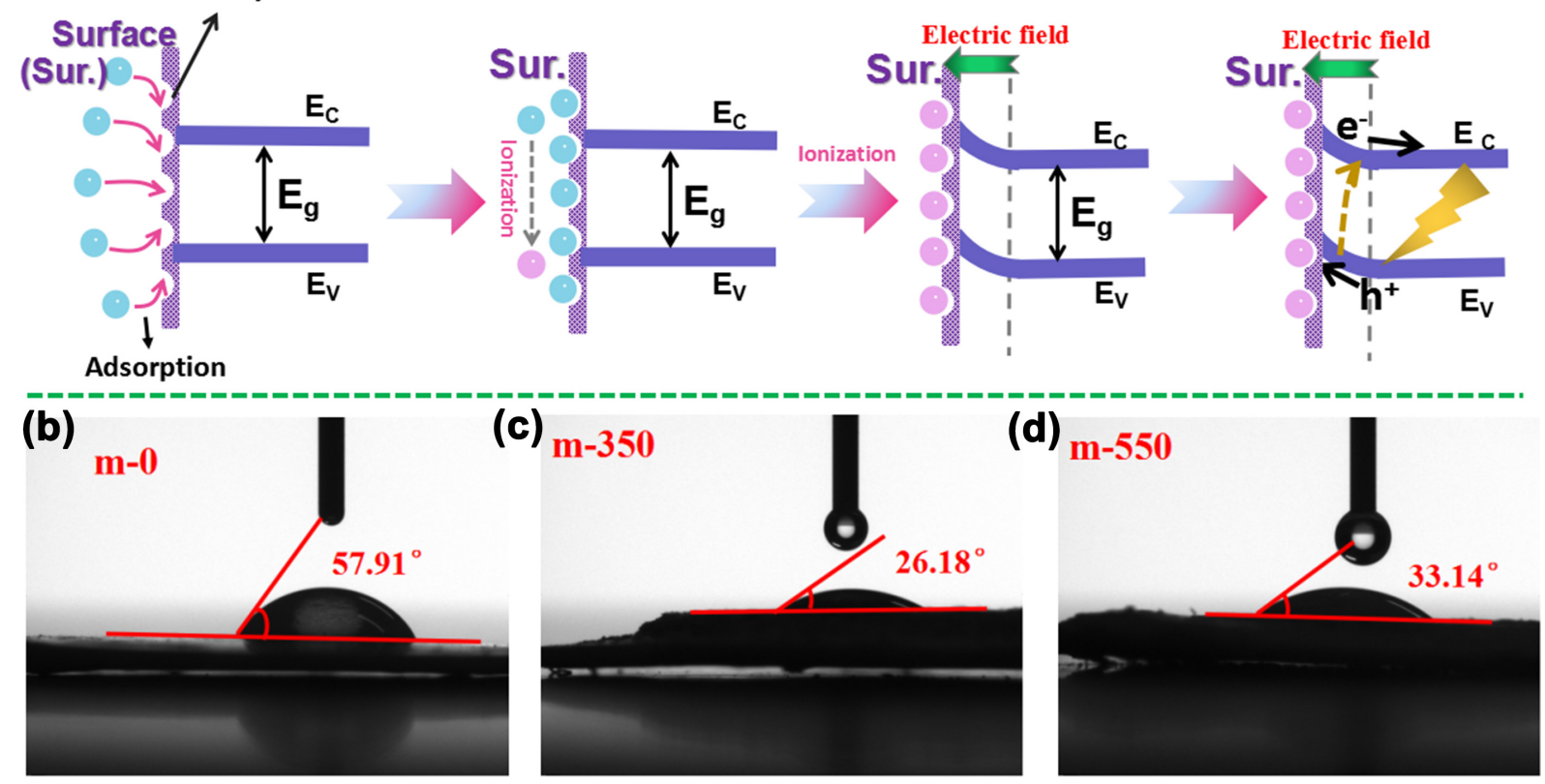

Figure 7. The effect of surface oxygen vacancy to the adsorption and energy bands of $m-350$ (a); contact angle image of $\mathrm{m}-0$ (b); contact angle image of $\mathrm{m}-350$ (c); contact angle image of m-550 (d).
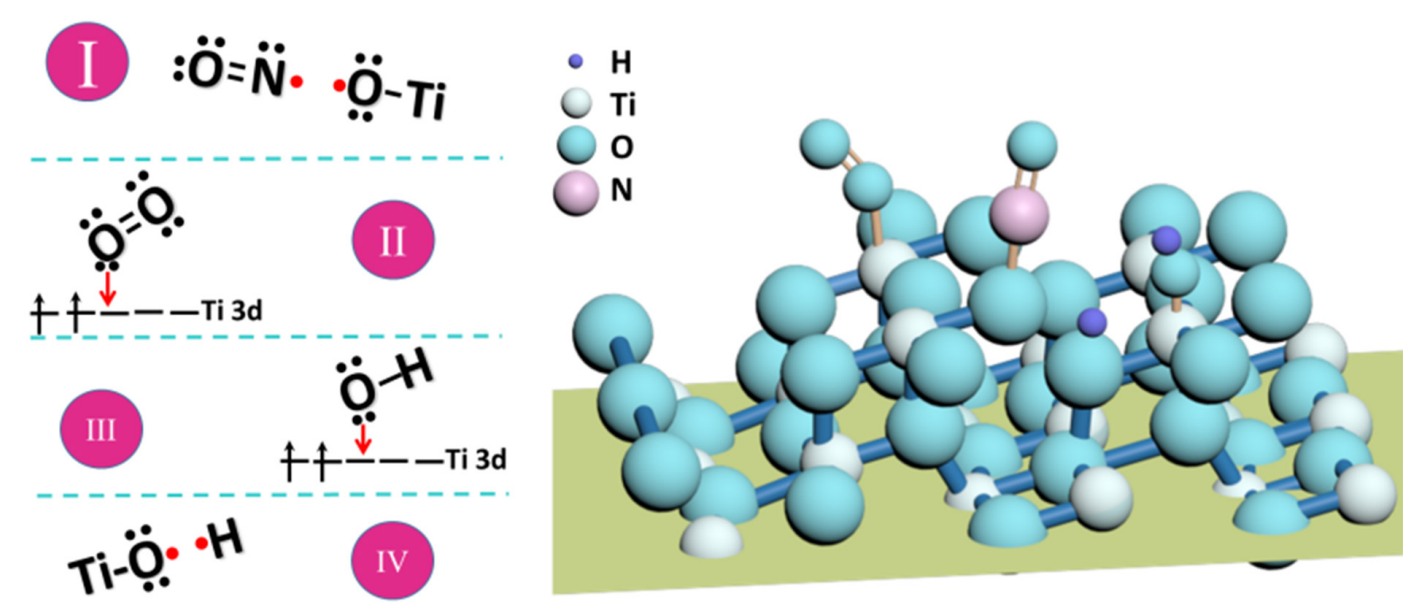

Scheme 1. The adsorption of adsorbates on the surface of $\mathrm{TiO}_{2}$.
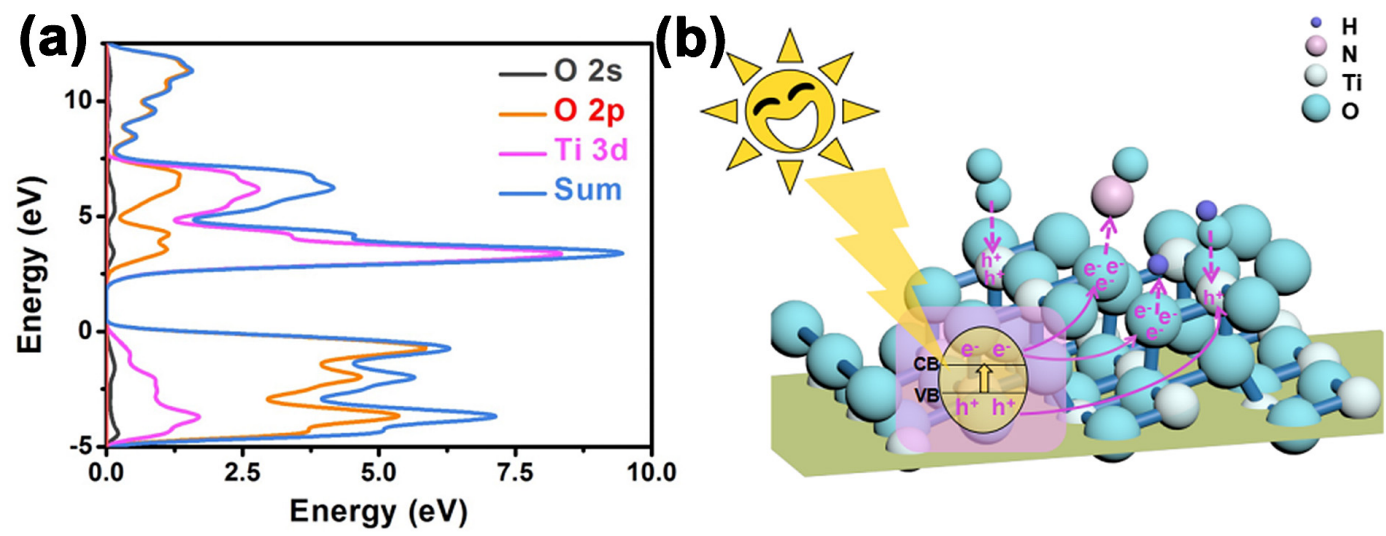


\section{WILEY-VCH}

Figure 8. Density of states (DOS) pattern of $\mathrm{TiO}_{2}(\mathrm{a})$; the diagram of redox reaction on the surface of $\mathrm{TiO}_{2}(\mathrm{~b})$.

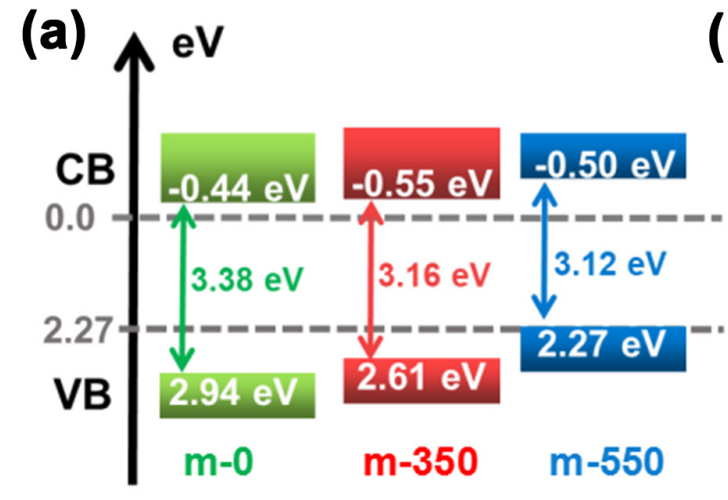

(b)

(c)

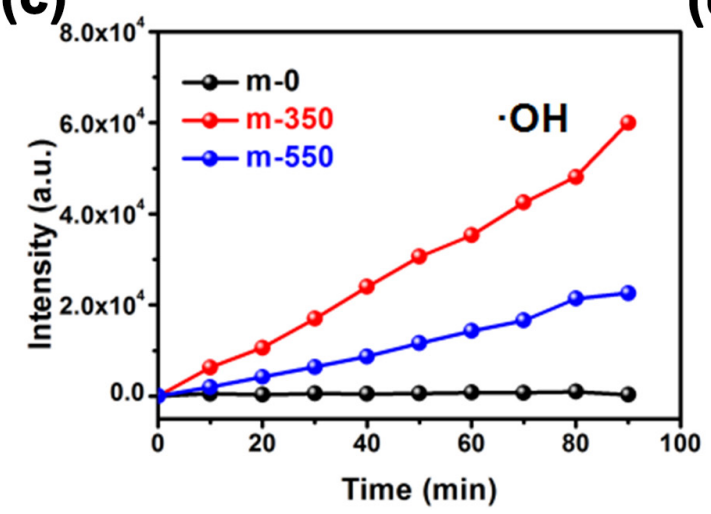

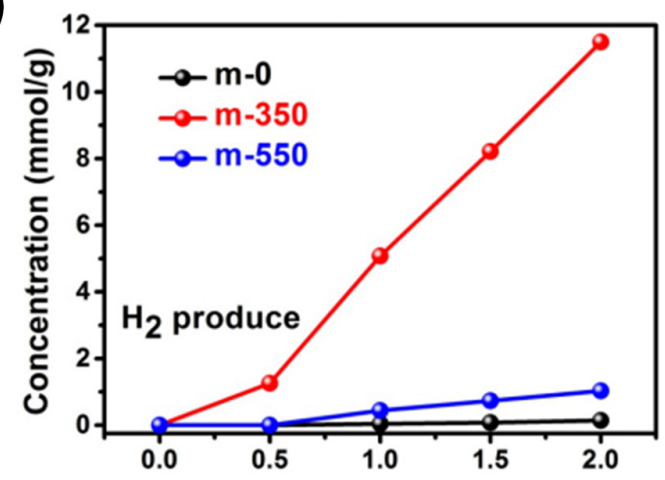

(d)

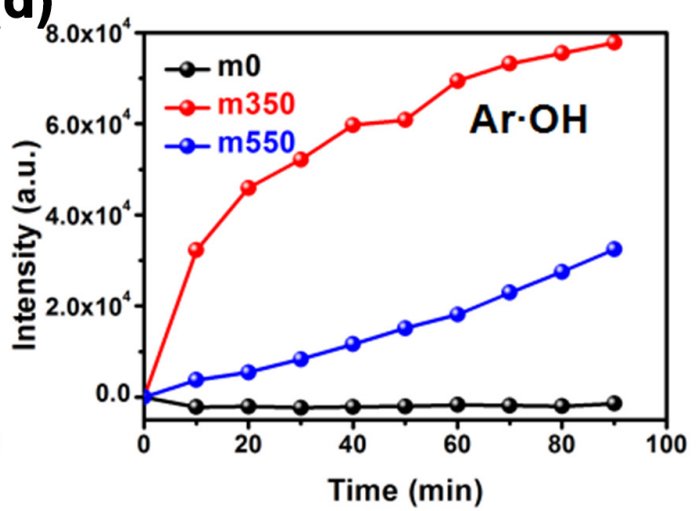

Figure 9. The diagram of energy bands of different samples (a); $\mathrm{H}_{2}$ evolution ability of the different samples (b); the hydroxyl radical production abilities of different samples (c); the hydroxyl radicals production abilities of different samples under anaerobic environment (d).

Table 1. The results of positron annihilation experiments; $\tau_{1}$ and $\tau_{2}$ are positron lifetimes; $I_{1}$ and $I_{2}$ are concentrations of $\tau_{1}$ and $\tau_{2}$. (The data were carried out with a fast-fast coincidence ORTEC system with a time resolution of $\sim 200 \mathrm{ps}$. A $30 \mu \mathrm{Ci}$ source of ${ }^{22} \mathrm{Na}$ was sandwiched between two identical samples, and the total count was 3 million. All the spectra were analyzed by the LIFETIME9 program.)

\begin{tabular}{ccccc}
\hline Samples & $\tau_{1} / \mathrm{ns}$ & $\mathrm{I}_{1}$ & $\tau_{2} / \mathrm{ns}$ & $\mathrm{I}_{2}$ \\
\hline $\mathrm{m}-0$ & 0.290 & $33.70 \%$ & 0.356 & $66.30 \%$ \\
$\mathrm{~m}-350$ & 0.317 & $95.97 \%$ & 0.510 & $4.03 \%$ \\
$\mathrm{~m}-550$ & 0.292 & $74.96 \%$ & 0.473 & $25.04 \%$ \\
\hline
\end{tabular}




\section{WILEY-VCH}

The medium-degree crystallinity $\mathrm{TiO}_{2}$ sample mainly containing surface O-vacancies (in-situ introduced) exhibits the excellent NO removal activity. The systematic study of photocatalytic mechanism demonstrates that the surface $\mathrm{O}$-vacancies significantly promote the adsorption of $\mathrm{H}_{2} \mathrm{O}$ molecules and improve charge transfer to the adsorbed $\mathrm{H}_{2} \mathrm{O}$ forming $\cdot \mathrm{OH}$, thus dramatically enhancing the photocatalytic NO removal activity.

Nitric oxide removal, oxygen vacancies, photocatalysis, $\mathrm{TiO}_{2}$

Xiaoling Shen, Guohui Dong*, Lan Wang, Liqun Ye, and Jianwu Sun*

Enhancing Photocatalytic Activity of NO Removal Through an In-Situ Control of Oxygen Vacancies in Growth of $\mathrm{TiO}_{2}$

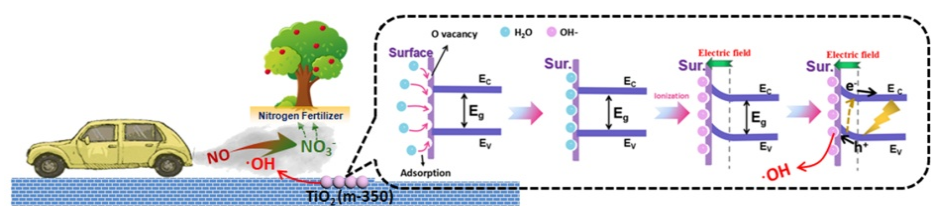




\section{WILEY-VCH}

Copyright WILEY-VCH Verlag GmbH \& Co. KGaA, 69469 Weinheim, Germany, 2016.

\section{Supporting Information}

\section{Enhancing Photocatalytic Activity of NO Removal through an in-situ Control of Oxygen Vacancies in growth of $\mathrm{TiO}_{2}$}

Xiaoling Shen, Guohui Dong*, Lan Wang, Liqun Ye, and Jianwu Sun*

\section{S1. Experimental Procedures}

\section{S1.1 Photocurrent experiment}

Photocurrent experiments were performed on the $\mathrm{CHI} 660 \mathrm{E}$ electrochemistry workstation by the three-electrode method. ${ }^{[1,2]}$ In experiments, $\mathrm{NaSO}_{4}$ solution $(0.1 \mathrm{M})$ was used as electrolyte. Calomel electrode and platinum plate were chosen as reference electrode and counter electrode, respectively. Working electrode was fabricated based on a $2 \times 1.5 \mathrm{~cm}^{2}$ $\mathrm{SnO}_{2}: \mathrm{F}$ (FTO) glass. The fabrication process as follow: $30 \mathrm{mg}$ samples were suspended in 10 $\mu \mathrm{l}$ absolute ethyl alcohol by ultrasonic dispersion in a $2 \mathrm{~mL}$ centrifuge tube. Then, the suspension was uniformly coated on the conducting surface of FTO glass via drop-coating technique. Subsequently, the electrode sheets were dried under the infrared irradiation. Visible light $(\lambda=420 \mathrm{~nm})$ was provided by a $300 \mathrm{~W}$ Xe lamp with a $420 \mathrm{~nm}$ band pass filter.

\section{S1.2 Detection of $\cdot \mathbf{O H}$}

\section{S1.2.1 $\cdot$ OH test at liquid-solid interface}

The concentration of $\mathrm{OH}$ generated in aerobic solution was measured by using terephthalic acid $(0.005 \mathrm{~mol} / \mathrm{L})$ as a fluorescent reagent. ${ }^{[3,4]}$ This detection experiment was carried out in a $150 \mathrm{~mL}$ glass beaker, which contains $100 \mathrm{~mL}$ fluorescent reagent and $100 \mathrm{mg}$ samples. Then, this beaker was illuminated with visible light $(\lambda=420 \mathrm{~nm})$ under continuous stirring. In illumination process, $4 \mathrm{~mL}$ of suspensions were taken from beaker every $10 \mathrm{~min}$. After centrifugation, the fluorescence spectrum of supernatant was measured under the excitation wavelength of $312 \mathrm{~nm}$. The scan range of fluorescence spectrum is $330-600 \mathrm{~nm}$. 


\section{WILEY-VCH}

The concentration of $\cdot \mathrm{OH}$ generated in anaerobic solution was measured by the same procedure, but the dissolved oxygen was eliminated by continuous blowing of high purity argon gas.

\section{S1.2.2 $\cdot$ OH test at gas-solid interface}

To detect the generated $\cdot \mathrm{OH}$ in the gas-solid interface, $50 \mathrm{mg}$ of samples and $0.1 \mathrm{~g}$ of terephthalic acid were first mixed uniformly. Then, the mixture samples were uniformly paved in a sample dish (Radius $=3 \mathrm{~cm}$ ), and it was irradiated by the visible light $(\lambda=420 \mathrm{~nm})$ from the top down. For comparison, the other sample dish with the same mixture samples was placed in dark condition to be used as the control experiment. After 30 minutes of the reaction, the mixtures were washed by $1 \mathrm{ml}$ of water through the ultrasonic method. The supernatant was collected by centrifugation, and this step was repeated 5 times to ensure that all the reaction products of terephthalic acid and produced $\cdot \mathrm{OH}$ dissolved in the supernatant. Finally, the fluorescence spectrum of supernatant was measured under the excitation wavelength of $312 \mathrm{~nm}$. The scan range of fluorescence spectrum is 330-600 nm.

\section{S1.3 Scavenging Experiments}

To confirm the role of $\cdot \mathrm{OH}$ and holes $\left(\mathrm{h}^{+}\right)$, tert-butanol (TBA) and potassium iodide (KI) were chosen as scavenger of $\cdot \mathrm{OH}$ and $\mathrm{h}^{+}$, respectively. ${ }^{[5,6]}$ The experimental process was as follows: (1) $50 \mathrm{mg}$ of sample with one kind of scavenger (TBA: $0.1 \mathrm{mM}$ or KI: $0.1 \mathrm{~g}$ ) was added into $10 \mathrm{~mL}$ of distilled $\mathrm{H}_{2} \mathrm{O}$; (2) the mixture suspension was ultrasonicated for $20 \mathrm{~min}$ and then coated onto the sample dish $(\mathrm{R}=3 \mathrm{~cm})$; (3) the coated dish was dried at $60{ }^{\circ} \mathrm{C}$ until the water was completely removed; (4) the sample dish was used in the NO removal experiments.

\section{S1.4 The density of functional theory (DFT) calculation methods}




\section{WILEY-VCH}

All the theoretical calculations were carried out by DFT calculations with the Generalized gradient approximation (GGA) and Perdew-Burke-Ernzerhof (PBE) exchangecorrelation energy functional. We build a $2 \times 2 \times 1$ super-cell. The energy and force converged to $10^{-5} \mathrm{eV} /$ atom and $0.05 \mathrm{eV} / \AA$. The kinetic energy cutoff of the system was $400 \mathrm{eV}$.

\section{S2. Results and Discussion}

\section{S2.1 XPS Analysis}

From Figure 1b and 1c, the XPS patterns of all the samples are similar. In detail, the Ti $2 \mathrm{p}$ peaks at about 456.86 and $462.56 \mathrm{eV}$ correspond to the $2 \mathrm{p} 3 / 2$ and $2 \mathrm{p} 1 / 2$ of $\mathrm{Ti}^{4+}$, respectively. ${ }^{[7]}$ All the O 1s XPS patterns of three samples can be divided into three peaks at 527.8, 530.1 and $531.5 \mathrm{eV}$, which correspond to the $\mathrm{O}$ atom in Ti-O-Ti, Ti-OH and adsorbed $\mathrm{H}_{2} \mathrm{O}$ molecular, respectively. ${ }^{[8]}$ Above results confirm that the composition of $\mathrm{m}-0$ is $\mathrm{TiO}_{2}$.

\section{S2.2 Calculation of the $\mathrm{N}$ balanced}

In the Figure 2d, the concentration of $\mathrm{NO}_{3}{ }^{-}$detected by ion chromatography in the washing solution of used $\mathrm{m}-0, \mathrm{~m}-350$, and $\mathrm{m}-550$ were $0.25,18.7$, and $0.85 \mu \mathrm{g} / \mathrm{L}$, respectively.

A. The computational formula of the consumption of $\mathrm{NO}\left(\mathrm{C}_{\mathrm{NO}}\right)$ is $C_{N O}=\sum_{t=1}^{t=30} N O c$; the results in $\mathrm{m}-0, \mathrm{~m}-350$ and $\mathrm{m}-550$ were $1.63,7.24$ and $0.35 \mathrm{ppm}$, respectively. The computation formula of concentration of consumed NO $\left(\mathrm{C}_{\mathrm{NO}}\right)$ is $C_{N O}=\frac{30 \times C_{N O}}{22.4}$. The results of m- 0 , m-350 and $\mathrm{m}-550$ were $2.18,9.70$, and $0.47 \mu \mathrm{g} / \mathrm{L}$, respectively.

B. The generated $\mathrm{NO}_{2}\left(\mathrm{C}_{\mathrm{NO} 2}\right)$ is: $C_{N O 2}=\sum_{t=1}^{t=30} N O G$, the results of $\mathrm{m}-0$ were $0.55,0$, and 0 ppm, respectively. The concentration of $\mathrm{NO}$ which converted to $\mathrm{NO}_{2}\left(\mathrm{C}_{\mathrm{NO} / \mathrm{NO} 2}\right)$ is 


\section{WILEY-VCH}

$C_{\mathrm{NO} / \mathrm{NO} 2}=\frac{30 \times C_{\mathrm{NO} 2}}{22.4}$; the results in $\mathrm{m}-0, \mathrm{~m}-350$, and $\mathrm{m}-550$ were $0.73,0$, and $0 \mu \mathrm{g} / \mathrm{L}$, respectively.

C. In this system, the concentration $\mathrm{NO}$ converted to $\mathrm{NO}_{3}{ }^{-}$(C $\left.\mathrm{C}_{\mathrm{NO} \text { NO3- }}\right)$ is: $\mathrm{C}_{\mathrm{NO} / \mathrm{NO}_{-}-}=\frac{30 \times \mathrm{C}_{\mathrm{NO} 3}-}{22.4}$. The results of $\mathrm{m}-0, \mathrm{~m}-350$ and $\mathrm{m}-550$ were $0.12,9.05$ and 0.41 $\mu \mathrm{g} / \mathrm{L}$, respectively.

D. The values of $\mathrm{C}_{\mathrm{NO} \text { NO3- }}+\mathrm{C}_{\mathrm{NO} \text { NO2 }}$ in $\mathrm{m}-350$ and $\mathrm{m}-550$ systems were 9.05 and $0.41 \mu \mathrm{g} / \mathrm{L}$, respectively. These two values are similar to the $C_{N O}$ values of $m-350$ and $m-550$, suggesting that the formed $\mathrm{NO}_{3}{ }^{-}$can meet with the consumed $\mathrm{NO}$ in $\mathrm{m}-350$ and $\mathrm{m}-550$ systems. The value of $\mathrm{C}_{\mathrm{NO} \text { NO3- }}+\mathrm{C}_{\mathrm{NO} \text { NO2 }}$ in $\mathrm{m}-0$ system is $0.62 \mu \mathrm{g} / \mathrm{L}$. This value is far less than the consumed $\mathrm{NO}$ in $\mathrm{m}-0$ system, indicating that the removal of $\mathrm{NO}$ in $\mathrm{m}-0$ system mainly depends on adsorption process.

\section{S2.3 Calculation of Desorption energy}

$Q_{a b s}=C_{p} m \Delta t$

In this formula, $\boldsymbol{Q}_{a b s}$ is the absorbed energy $(\mathrm{J}) ; \boldsymbol{C}_{\boldsymbol{p}}$ is the specific heat capacity of the NO gas $(\mathrm{J} / \mathrm{kg} \cdot \mathrm{K})$, The specific heat capacity of NO gas is $974.04 \mathrm{~J} /(\mathrm{kg} \cdot \mathrm{K}) ; \boldsymbol{m}$ is the mass $(\mathrm{kg})$ of $\mathrm{NO} ; \boldsymbol{\Delta t}$ is the temperature difference between $293 \mathrm{~K}$ and the desorption temperature; the desorption temperature of $\mathrm{m}-0, \mathrm{~m}-350$ are $433 \mathrm{~K}$ and $338 \mathrm{~K}$, respectively. The $\boldsymbol{Q}_{a b s}$ of $\mathrm{m}-0$ and $\mathrm{m}-350$ were calculated as $4.09 \mathrm{KJ} \cdot \mathrm{mol}^{-1}$ and $1.31 \mathrm{KJ} \cdot \mathrm{mol}^{-1}$, respectively.

\section{S2.4 Calculation of Apparent Quantum Yield}

\section{A: The incident radiant flux $(P)$}

$$
P=E A
$$

Where $\boldsymbol{A}$ is the irradiation area $(R=3 \mathrm{~cm})$ during the photocatalytic reaction, $28.26 \mathrm{~cm}^{2}$. The result of $\boldsymbol{P}$ in the system is $2.10 \mathrm{~W}$. where $\boldsymbol{E}$ is the average irradiation; the value is 


\section{WILEY-VCH}

measured by the light power meter (PLS-MW2000, Perfectlight). It was measured by the light power meter (PLS-MW2000, Perfectlight), and the value is $741.51 \mathrm{~W} \cdot \mathrm{m}^{-2}$.

\section{B: The number of incident photons $\left(N_{p}\right)$}

$$
N_{p}=\frac{P \lambda t}{h c}
$$

Where, $t$ is the illumination time (1800 seconds), $\lambda$ is the wavelength of the incident light $(420 \mathrm{~nm}), \boldsymbol{h}$ is the Planckconstant $\left(6.62 \times 10^{-34} \mathrm{~J} \cdot \mathrm{s}\right)$ and $\boldsymbol{c}$ is the velocity of light $(3.0 \times 108$ $\left.\mathrm{m} \cdot \mathrm{s}^{-1}\right) .{ }^{9}$ The result of $N_{\boldsymbol{P}}$ was calculated as $7.89 \times 10^{21}$.

\section{C: The apparent quantum yield $(\psi)$.}

$$
\psi=\frac{n i N_{A}}{N_{p}}
$$

In this formula, $\boldsymbol{n}$ is the mole number of $\mathrm{NO}_{3}{ }^{-} ; \boldsymbol{i}$ means the required charger quantity during the NO removal process; $\boldsymbol{N}_{\boldsymbol{A}}$ is the Avogadro constant $\left(6.02 \times 10^{23}\right) .{ }^{10}$ The concentration of $\mathrm{NO}_{3}{ }^{-}\left(\mathrm{C}_{\mathrm{NO}}-\right)$ detected by ion chromatography in the washing solution of used $\mathrm{m}-0, \mathrm{~m}-350$ and $\mathrm{m}-550$ were $0.25,18.7$ and $0.85 \mu \mathrm{g} / \mathrm{L}$, respectively. Therefore, the mole number of $\mathrm{NO}_{3}{ }^{-}$production of $\mathrm{m}-0, \mathrm{~m}-350$ and $\mathrm{m}-550$ were $2.24 \times 10^{-9}, 1.68 \times 10^{-7}$, and 7.62 $\times 10^{-9}$ mol, respectively. According to equations $1-5$, the generation of one $\mathrm{NO}_{3}{ }^{-}$requires three $\mathrm{h}^{+}$. Therefore, the value of $\boldsymbol{i}$ is 3 .

The apparent quantum yield $(\psi)$ of m-0, m-350 and m-550 were calculated as $0.91 \%$, $68.33 \%$ and $3.11 \%$ respectively. 


\section{WILEY-VCH}

\section{S3. Figures}
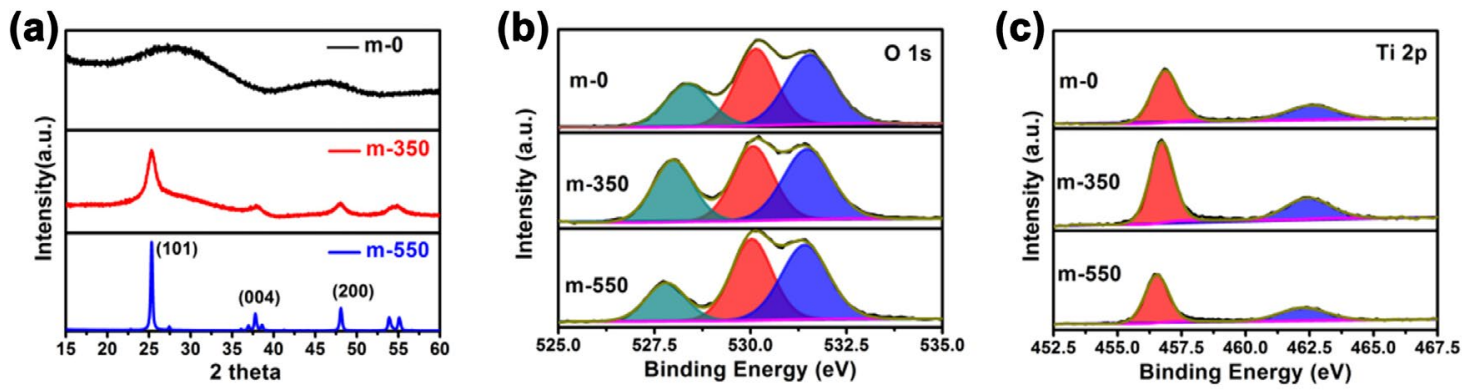

Figure S1. The XRD patterns (a), the O 1s XPS spectra (b), and the Ti 2p XPS spectra (c) of $\mathrm{TiO}_{2}$ samples without $(\mathrm{m}-0)$ and with calcination at $350{ }^{\circ} \mathrm{C}(\mathrm{m}-350)$ and $550{ }^{\circ} \mathrm{C}(\mathrm{m}-550)$.

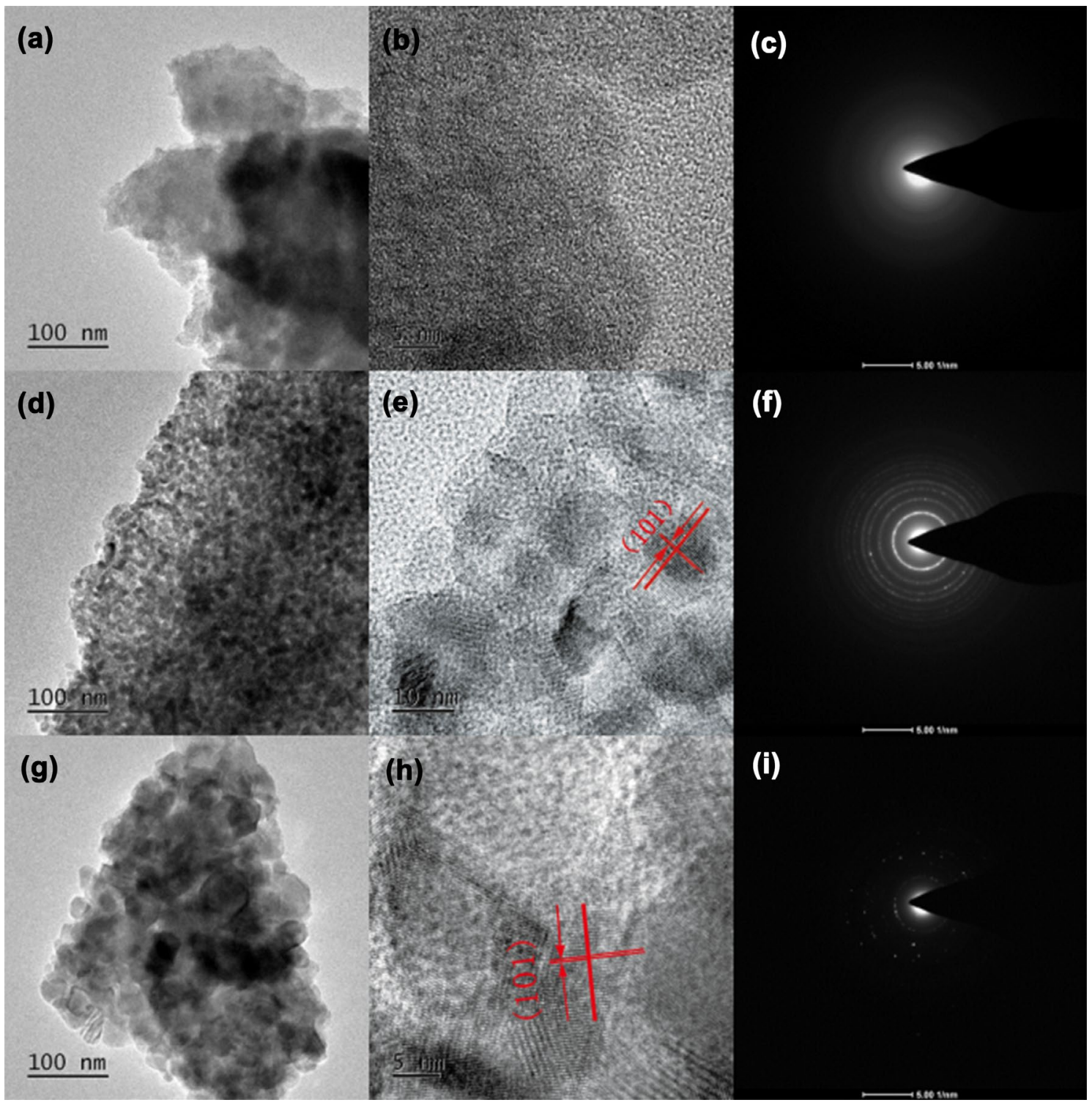

Figure S2. TEM image (a), HRTEM image (b), and SAED pattern (c) of m-0; TEM image (d), HRTEM image (e), and SAED pattern (f) of m-350; TEM image (g), HRTEM image (h), and SAED pattern (i) of $\mathrm{m}-550$. 


\section{WILEY-VCH}

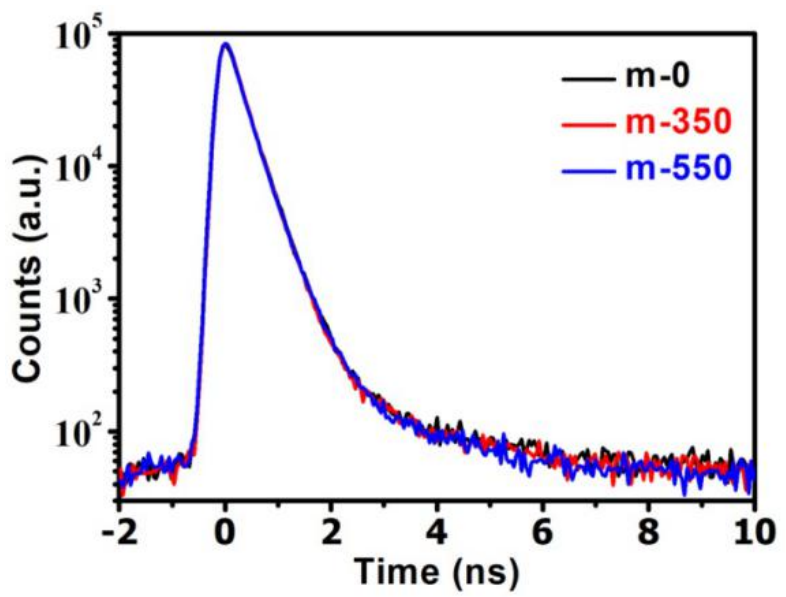

Figure S3. The positron annihilation spectra of m-0, m-350, and m-550 samples.
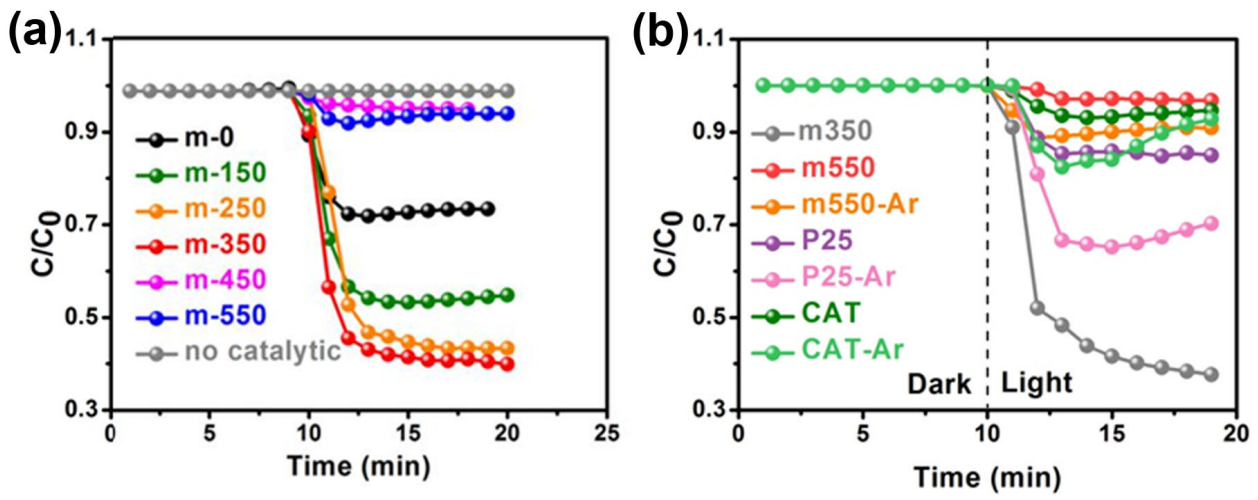

Figure S4. Photocatalytic NO removal activities of different samples in the optimization experiment (a) and the similar sample comparison experiments (b).
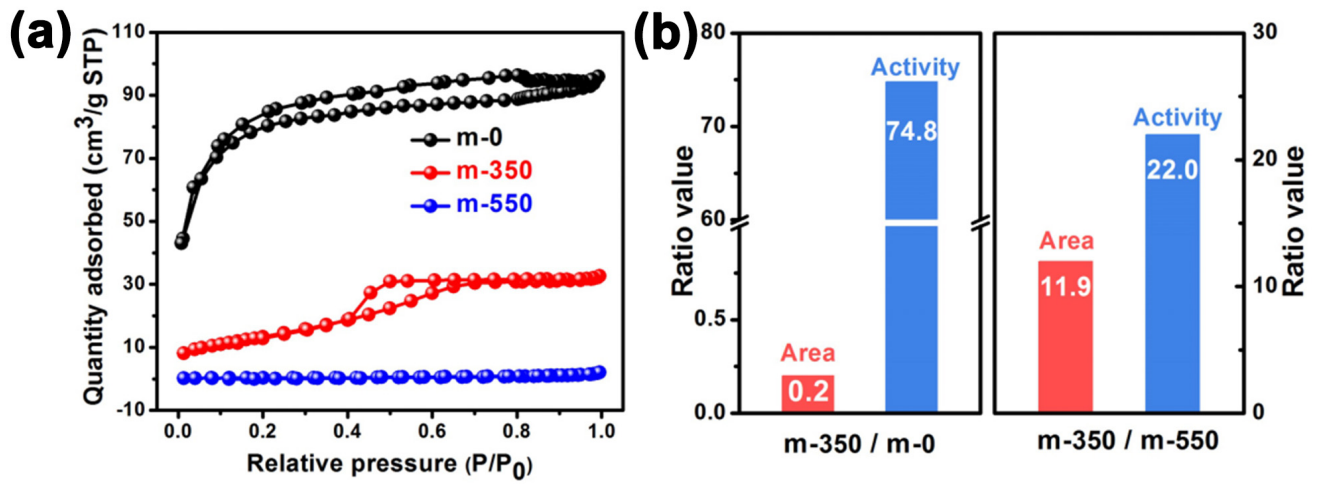

Figure S5. $\mathrm{N}_{2}$ adsorption desorption isotherms of $\mathrm{m}-0, \mathrm{~m}-350$, and $\mathrm{m}-550$ (a); the ratio values of areas and activities between $\mathrm{m}-350$ and $\mathrm{m}-0$ (b). 


\section{WILEY-VCH}

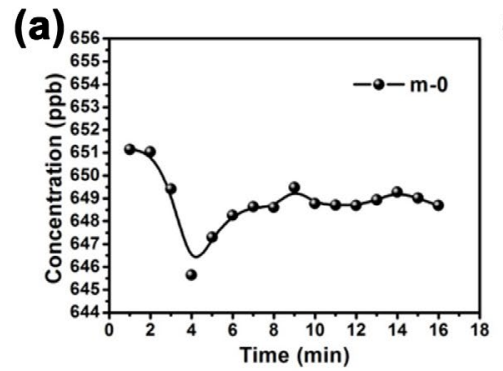

(b)

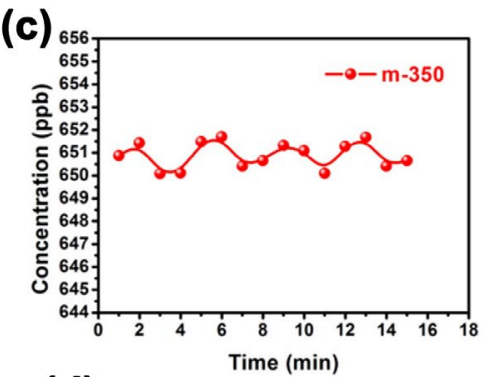

(d)

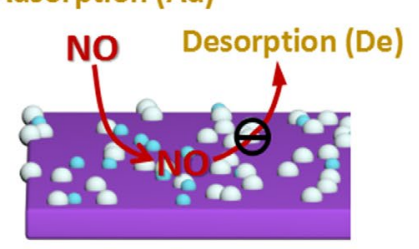

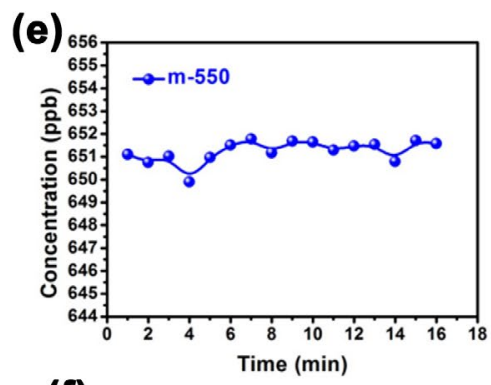

(f)

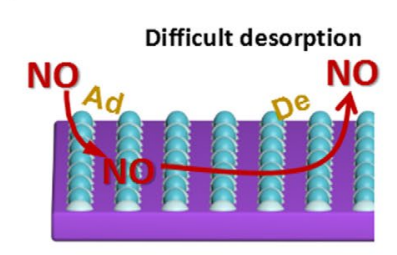

Figure S6. The NO adsorption quantity (a) and schematic diagram (b) of $\mathrm{m}-0$; the NO adsorption quantity (c) and schematic diagram (d) of m-350; the NO adsorption quantity (e) and schematic diagram (f) of m-550.

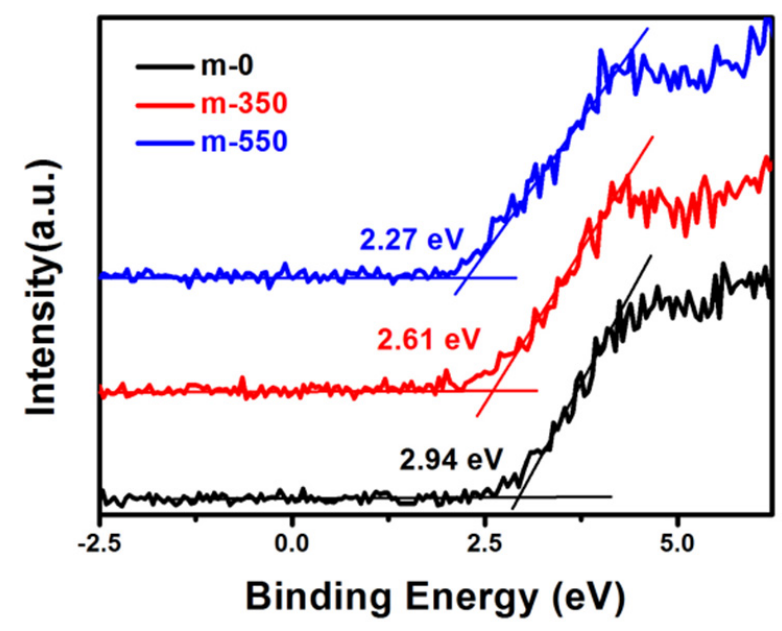

Figure S7. The VB-XPS spectra of m-0, m-350, and m-550 samples. 
(a)

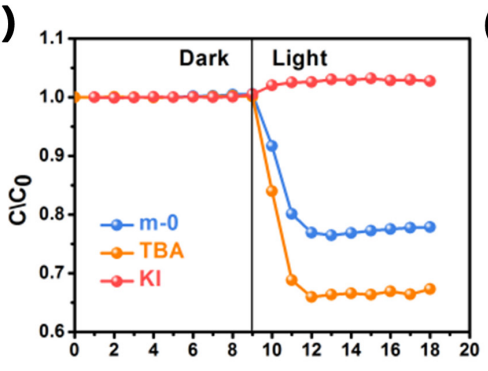

(d)

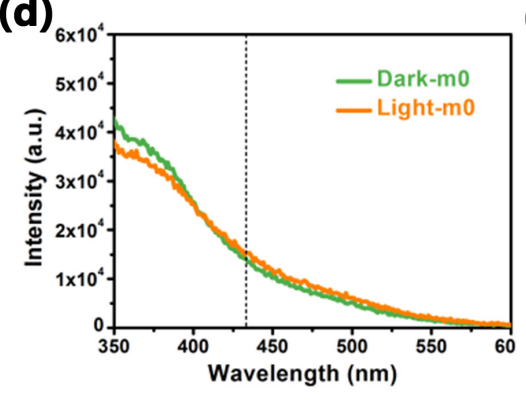

(b)

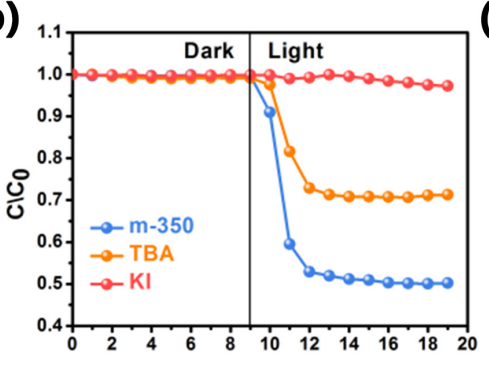

(e)

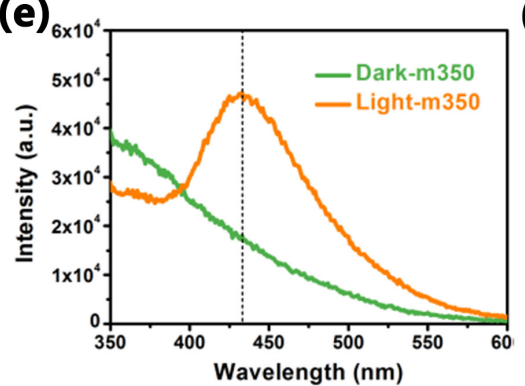

(c)

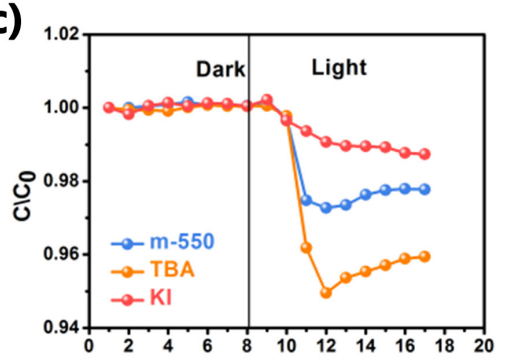

(f)

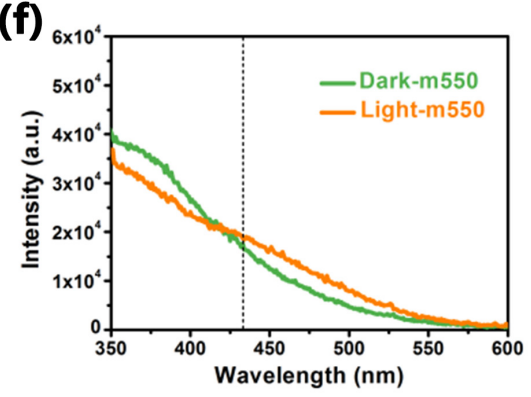

Figure S8.The trapping experiments of $\mathrm{m}-0(\mathrm{a}), \mathrm{m}-350(\mathrm{~b})$, and $\mathrm{m}-550$ (c); the production of hydroxyl radical in gas-solid phase condition of m- 0 (d); m-350 (e); m-550 (f).
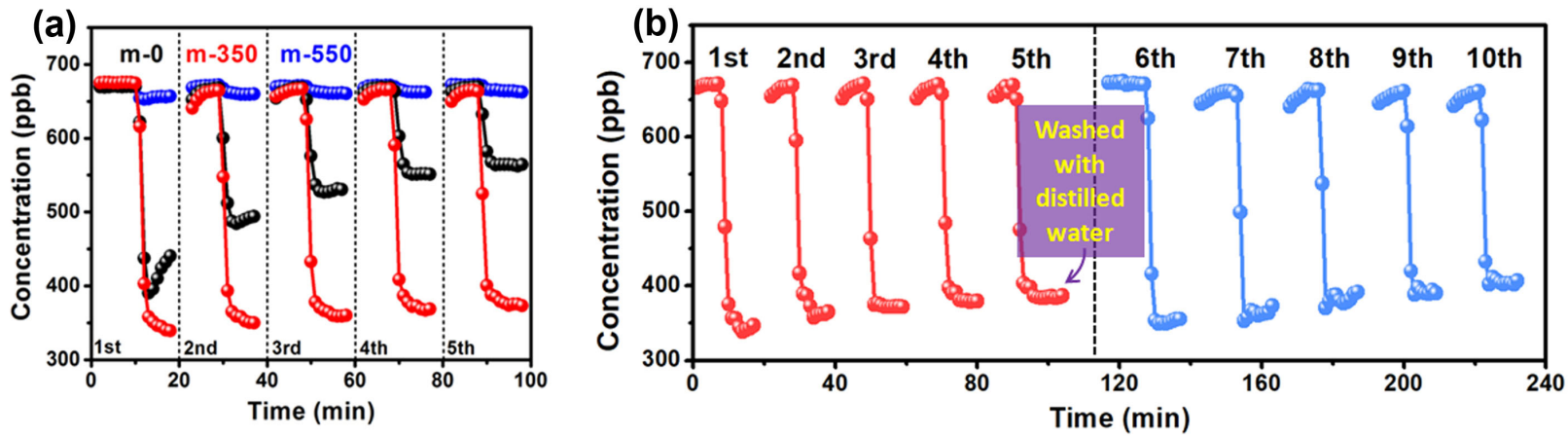

Figure S9. The reusability tests of $\mathrm{m}-0, \mathrm{~m}-350$ and $\mathrm{m}-550$ (a); the reusability tests of $\mathrm{m}-350$ (b).

(a)

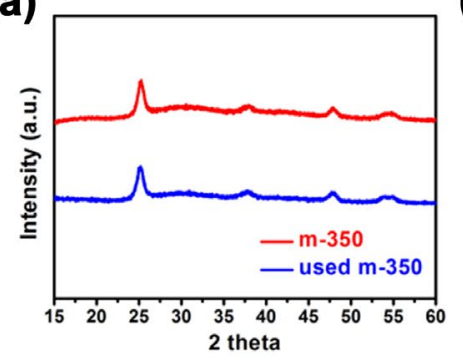

(b)

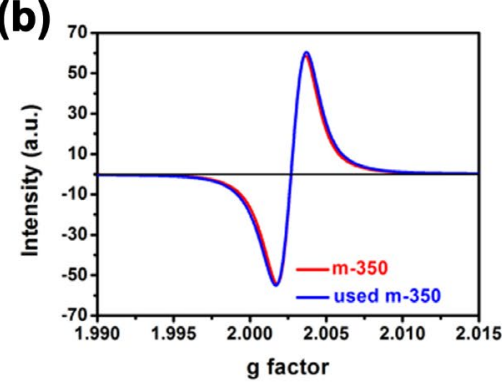

(c)

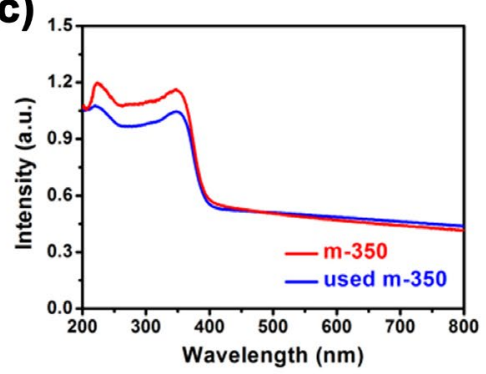

Figure S10. The XRD patterns (a), EPR patterns (b), and UV-vis absorption spectra (c) of the pristine $\mathrm{m}-350$ and used $\mathrm{m}-350$.

\section{S4. References}




\section{WILEY-VCH}

[1] J. Q. Zhang, Z. P. Xing, J. Y. Cui, Z. Z. Li, S. Y. Tan, J. W. Yin, J. L. Zou, Q. Zhu, W. Zhou, Dalton Transactions, 2018, 47, 4877.

[2] S. N. Li, G. H. Dong, R. Hailili, L. P. Yang, Y. X. Li, F. Wang, Y. B. Zeng, C. Y. Wang, Appl. Catal. B: Environ. 2016, 190, 26.

[3] Y. X. Qin, F. H. Song, Z. H. Ai, P. P. Zhang, L. Z. Zhang, Environ. Sci. Technol. 2015, 49, 7948.

[4] Y. Nosaka, S. Komori, K. Yawata, T. Hirakawa, A. Y. Nosaka, (2003). Phys. Chem. Chem. Phys. 2003, 5, 4731.

[5] G. H. Dong, L. P. Yang, F. Wang, L. Zang, C. Y. Wang, ACS Catalysis, 2016, 6, 6511.

[6] L. L. Zhao, G. H. Dong, L. Zhang, Y. F. Lu, Y. Huang, ACS Appl. Mater. Interfaces, 2019, 11, 10042.

[7] J. Shao, W. C. Sheng, M. S. Wang, S. J. Li, J. R. Chen, Y. Zhang, S. S. Cao, Appl. Catal., B-Environ. 2017, 209, 311.

[8] N. Kruse, S. Chenakin, Appl. Catal. A: Gen. 2011, 391, 367.

[9] T. F. Yeh, S. J. Chen, H. Teng, Nano Energy. 2015, 12, 476.

[10] M. Y. Xing, J. L. Zhang, B. C. Qiu, B. Z. Tian, M. Anpo, M. Che, Small. 2015, 11, 1920. 